\title{
Effects of heterogeneity, connectivity and density variations on mixing and chemical reactions under temporally fluctuating flow conditions and the formation of reaction patterns
}

\author{
Maria Pool ${ }^{1,2}$ and Marco Dentz ${ }^{1,2}$ \\ ${ }^{1}$ Spanish National Research Council (IDAEA-CSIC), Barcelona, Spain \\ ${ }^{2}$ Associated Unit: Hydrogeology group (UPC-CSIC), Barcelona, Spain
}

Key Points:

- The coupling of transient forcing and heterogeneity leads to strong local enhancement of mixing and reactivity.

- Buoyancy forces cause a compression of the interface which in turn increases the mixing and reaction efficiency.

- These mechanism provide a plausible explanation for the formation of dissolution patterns.

Corresponding author: Maria Pool, mpoolr@gmail.com 


\section{Abstract}

Solute mixing, spreading and fast chemical reactions in aquifers are strongly influenced by spatial variability of the hydraulic properties, temporal flow fluctuations and fluid density differences. We study the coupling of heterogeneity, transient forcing and density-driven flow on mixing and chemical reactions between two fluids of different density under a stable stratification. We consider the reaction of the fast dissolution of calcite. We find that temporal fluctuations and heterogeneity cause strong local enhancement of the mixing and reaction rates and this impact increases with the degree of connectivity of hydraulic conductivity. The global mixing and reactivity, however, are on the order of or smaller than their homogeneous counterparts due to heterogeneity-induced fluid segregation. The local maxima of the mixing and reaction rates are found to be located around strongly stretched regions corresponding to high velocity zones where dispersive mass transfer mechanisms are increased by dispersion. We also find that density variations compress the interface, which in turn emphasizes local maxima in mixing and reaction rates. Numerical results provide evidence that the stretching of the interface induced by spatial heterogeneity and transient effects coupled with density variations leads to the formation of complex patterns of reactive hotspots, zones of enhanced reaction efficiency, and that its distribution is directly linked to the deformation properties and topology of the flow field. These results provide new insights into the role of spatial and temporal variability on the mixing and reaction efficiency as well as the formation of reactive geochemical patterns in actual environmental systems.

\section{Introduction}

Mixing and dispersion of solutes and induced reactive processes occurring in flow through porous media at different scales have recently received increasing attention because they play a key role in a number of important applications, such as groundwater management, aquifer remediation, artificial recharge and risk assessment in geological hazardous waste storage. While substantial progress has been made to characterize flow and solute transport processes, addressing conservative mixing and reactive transport in real-world environments remains a largely open question due to the inherent complexity of natural flow and transport systems.

Solute spreading and mixing are strongly affected by spatially nonuniform variations of the flow field in response to spatial heterogeneity of the hydraulic properties of 
the subsurface. Significant research has been devoted to quantify the impact of heterogeneity on the spatial extent of transported solute plumes [Gelhar, 1993; Dagan, 1987; Rubin, 2003], non-Fickian large scale transport [Berkowitz et al., 2006; Neuman and Tartakovsky, 2009], and the impact of heterogeneity on solute mixing and chemical reaction [Dentz et al., 2011]. Recently, the mechanisms of mixing in heterogenous porous media have been related to the deformation properties of the flow field through the stretching and coalescence of material elements [Villermaux, 2012; Le Borgne et al., 2015]. Heterogeneous flow fields are characterized by the emergence of connected high velocity channels which form braided networks of preferential flow paths. The resulting connectivity structure, which mainly depends on the log-conductivity variance and the dimensionality of the system [Fiori and Jankovic, 2012], dramatically influences flow and solute transport in aquifers [Fogg et al., 1998, 2000; Le Borgne et al., 2010; Bianchi et al., 2011; Pedretti et al., 2013; Willmann et al., 2008]. Thus, accurate characterization of channeling is essential to analyze flow and transport processes in porous media and intensive research efforts over the last decades have been devoted to define connectivity indicators [Zinn and Harvey, 2003; Knudby and Carrera, 2005; Renard and Allard, 2013] and delineate preferential flow paths in groundwater systems [Tyukhova et al., 2015; Tyukhova and Willmann, 2016]. Although these previous investigations provide invaluable insights into the role of heterogeneity and connectivity on the dispersion and mixing dynamics, they consider steady uniform flow and rarely incorporate the influence of transient effects.

Many real systems are strongly influenced by transient effects on multiple time scales, such as diurnal variations in evapotranspiration or seasonal pumping, recharge and droughts. Numerous studies have investigated the impact of temporal flow fluctuations on solute transport of contaminant plumes. However, a common approach used is to assume quasistationarity of the flow field, this means disregarding storativity, and constant dispersion. Under such conditions, a key finding has been that transient forcing parallel to the main flow direction has little influence on mixing, whereas transverse temporal fluctuations enhance transverse dispersion and reduce longitudinal dispersion [Rehfeldt and Gelhar, 1992; Dagan et al., 1996; Dentz and Carrera, 2003; Cirpka and Attinger, 2003; de Dreuzy et al., 2012]. For homogeneous porous media with velocity-dependent dispersion transient forcing leads to an important increase in transverse dispersion whereas longitudinal dispersion is reduced [Kinzelbach and Ackerer, 1986; Goode and Konikow, 1990]. Furthermore, Pool et al. [2016] derived an effective time-average formulation to describe the coupling be- 
tween mixing and oscillatory transient flow under nonstationary flow conditions for finite storativity. These authors demonstrated analytically, numerically and experimentally for homogeneous aquifers that temporal fluctuations of the flow conditions lead to a complex space and time-dependent flow response which induces enhanced spreading and mixing of dissolved substances. The systematic investigation of the interaction of medium heterogeneity, temporal flow fluctuations under velocity-dependent dispersion is an outstanding question.

Another key property that may markedly impacts groundwater flow patterns and significantly affect transport of active and passive contaminants, their dispersion, mixing and reaction behaviour is fluid-density variations. Density-driven flow plays an important role in many environmental problems such as seawater intrusion, freshwater-brine interfaces, dense contaminant plume migration, transport of DNAPLs, salt lakes, heat in geothermal systems and $\mathrm{CO}_{2}$ sequestration [Diersch and Kolditz, 2002; Neufeld et al., 2010; Hidalgo et al., 2015]. In flow systems with variable density, heterogeneity and temporal fluctuations may significantly influence flow and transport over many length scales. In the literature, the research on the effects of heterogeneity on solute transport in variable density systems has been mainly focused on the analysis of unstable convective flows, where a dense fluid overlies less dense one [Schincariol and Schwartz, 1990; Schincariol et al., 1997; Schincariol, 1998; Simmons et al., 2001]. In this case, heterogeneity induces enhanced vertical mixing and spreading of the plume. On the other hand, for seawater intrusion problems heterogeneity affects the interface inland migration and leads to a significant widening of the mixing zone between fresh and saltwater [Schwarz, 1999; Held et al., 2005; Abarca, 2006; Kerrou and Renard, 2010; Pool et al., 2015]. Thus, the coupling of heterogeneity and density effects is expected to increase the mixing efficiency. For vertical front displacement under stable density stratification this is different. Under these flow conditions, Landman et al. [2007b] demonstrated that density variations smoothen the irregularities in the interface and cause the deformation of the front and then the mixing zone between the two fluids to decrease due to the competition between viscous forces induced by heterogeneity and buoyancy forces. Therefore, proper quantification of the competition between spatial heterogeneity and the non-linear nature of density-driven flow coupled with transient forcing on solute transport is a key to understanding and predicting the degree of local mixing and its impact on chemical reactions. 
Besides heterogeneity of the medium, temporal flow fluctuations and density variations of the fluid, solute transport in the subsurface is strongly affected by chemical reactions between dissolved chemicals (fluid-fluid) as well as between the dissolved chemicals and the solid matrix (fluid-solid). Mixing processes in porous media are particularly important for controlling chemical reactions [e.g., Rezaei et al., 2005; De Simoni et al., 2005; Tartakovsky et al., 2008; Cirpka et al., 2008; Dentz et al., 2011; de Anna et al., 2014a] and can be related to the deformation properties of the flow field [de Anna et al., 2014b; Le Borgne et al., 2014; Hidalgo et al., 2015; Bandopadhyay et al., 2017]. A wide variety of chemical reactions occur between fluids and fluids and solids in density-dependent flow systems, including calcite dissolution and precipation [Rezaei et al., 2005; Izgec et al., 2008], cation exchange [Appelo and Willemsen, 1987; Appelo, 1994; Giménez-Forcada, 2010], dissolution/precipitation of gypsum [Magaritz and Luzier, 1985; Stoessell et al., 1993; Gomis-Yagües et al., 1997], dolomitization [Müller and Fischbeck, 1973; Magaritz et al., 1980] and karstification [Plummer, 1975; Hanshaw and Back, 1979; Smart et al., 1988; Singurindy et al., 2004]. To date the interplay between temporal and spatial variability and density effects on mixing and dispersion of solutes and chemical pattern formation remain largely unexplored, despite its importance in controlling reaction rates and reactive transport processes for large-scale problems.

The objective of this study is to quantify the complex interactions between aquifer connectivity, transient-forcing and fluid-density effects on mixing and chemical reactions in heterogeneous porous media. To this end, we perform two-dimensional constant and variable-density flow and transport Monte Carlo simulations. Log-normally distributed random permeability fields assuming a Gaussian model are generated. In order to evaluate the impact of aquifer connectivity, more complex heterogeneous fields characterized by connected patterns of high and low conductivity are considered. We analyze the mechanisms of mixing by focusing on the measures of the interface width, as well as diagnostics which quantify the deformation of the interface. For the reactive transport system, we consider the irreversible reaction of calcite dissolution. To this end, we map the reactive transport problem onto a conservative one through the use of mixing ratios and conservative components [De Simoni et al., 2005, 2007]. Global and local reaction efficiency is evaluated with emphasis placed on the location and topology of hotspots, zones of enhanced reactivity. The reaction patterns formation is related to the deformation of material fluid elements in the heterogeneous flow field and specifically its stretching behavior. In 
this study, we provide a plausible explanation for the formation of geochemical reaction patterns observed in coastal karst aquifers. The enhanced understanding and quantification of the interplay between spatial and temporal variability and density-driven flow developed in this study is expected to provide new insights into mixing and reactive transport mechanisms which is essential for their diagnosis and prediction in heterogeneous subsurface environments.

\section{Problem Statement and Methodology}

We investigate the effect of heterogeneity and density variations on mixing and chemical reactions between two fluids (fresh and saline water) for a stable stratification in response to periodic temporal flow fluctuations. A fully saturated two-dimensional heterogeneous porous medium is considered with the $z$-axis pointing vertically upward, see Figure 1. Fluid mass conservation is given by the continuity equation [Voss, 1984],

$$
\rho S_{p} \frac{\partial p}{\partial t}=-\nabla \cdot \rho \mathbf{q}
$$

where $S_{p}=\phi \alpha_{w}+(1-\phi) \alpha_{s}\left[\mathrm{LT}^{2} \mathrm{M}^{-1}\right]$ is the specific pressure storativity, $\alpha_{w}\left[\mathrm{LT}^{2} / \mathrm{M}\right]$ the compressibility of water, $\alpha_{s}\left[\mathrm{LT}^{2} / \mathrm{M}\right]$ of the solid matrix, $\phi\left[\mathrm{L}^{3} \mathrm{~L}^{-3}\right]$ is porosity, $\rho\left[\mathrm{ML}^{-3}\right]$ fluid density and $\mathbf{q}\left[\mathrm{LT}^{-1}\right]$ Darcy velocity defined below. Using the equivalent freshwater head $h_{f}=p /\left(\rho_{f} g\right)+z$ with $\rho_{f}\left[\mathrm{ML}^{-3}\right]$ freshwater density and the Boussinesq approximation, this equation reads as

$$
S_{f} \frac{\partial h_{f}}{\partial t}=-\nabla \cdot \mathbf{q}
$$

where $S_{f}=\rho_{f} g S_{p}\left[\mathrm{~L}^{-1}\right]$ is specific storativity. The Darcy velocity $q$ is given by the Darcy equation, which reads in terms of equivalent freshwater head as

$$
\mathbf{q}=-k\left(\nabla h_{f}+\frac{\rho-\rho_{f}}{\rho_{f}} \hat{e}_{z}\right)
$$

with $k\left[\mathrm{LT}^{-1}\right]$ the hydraulic conductivity and $\hat{e}_{z}$ the unit vector in $z$-direction. The spatially variable hydraulic conductivity $k(x, z)$ is modeled as a spatial random field, which is generated from a multi-Gaussian random field $f(x, z)$. We consider both lognormally dis- 
tributed $k(x, z)=\exp [f(x, z)]$ as well as connected $k(x, z)$ fields, which are generated by the transformation outlined in Zinn and Harvey [2003]. Details are given in Section 2.3.

We consider flow scenarios with constant and variable fluid density. For the densitydependent flow scenarios, fluid density $\rho$ is assumed to be a linear function of the salt mass fraction $\omega$ (mass of dissolved salt per unit mass of fluid) given by $\rho=\rho_{f}(1+\beta c$ ), where $\beta$ is the buoyancy factor defined as $\beta=\left(\rho_{s}-\rho_{f}\right) / \rho_{s}$ with $\rho_{s}$ and $\rho_{f}$ the saline and freshwater densities, respectively, and $c$ is the mixing ratio between the two fluids defined as $c=\omega / \omega_{s}$, with $\omega_{s}$ the salt mass fraction of the saline water. Equation (1) is subject to a dynamic prescribed head assumed to be sinusoidal along the bottom boundary $(z=0)$, characterized by a single harmonic with a constant amplitude $(A)$ and period $(\tau)$, which simulates for example tidal fluctuations; the top boundary is assumed to be located far from the prescribed harmonic flow perturbation so that the gradient of head can be considered to be zero (no-flow boundary of both salt and water) [Pool et al., 2016]. This setup is characterized by a temporally mean flow of 0 . Furthermore, the amplitude of the flow fluctuations decreases exponentially fast with distance from the boundary [see, e.g., Jacob, 1950; Ferris, 1951]. For an equivalent homogeneous medium, for example, the characteristic distance is given by the inverse of the wave number $\mu=\sqrt{S_{f} \pi /\left(k_{g} \tau\right)}$, where $k_{g}$ is the geometric mean conductivity. Note that this implies, that even for a homogeneous medium, the flow field varies in space. Solute dispersion in transient flow in a homogenous medium under these conditions were studied in Pool et al. [2016].

The mixing ratio $c$ evolves according to the advection-dispersion equation [e.g., Pool et al., 2016]

$$
\phi \frac{\partial c}{\partial t}=-\mathbf{q} \cdot \nabla c+\nabla \cdot \mathbf{D}_{\mathbf{h}} \nabla c,
$$

where local mixing is represented by a velocity-dependent dispersion coefficient $\mathbf{D}_{\mathbf{h}}$ $\left[\mathrm{L}^{2} \mathrm{~T}^{-1}\right]$ defined as $\mathbf{D}_{\mathbf{h}}(x, z, t)=\mathbf{D}(x, z, t)+\phi \boldsymbol{I} D_{m}$, with $D_{m}\left[\mathrm{~L}^{2} \mathrm{~T}^{-1}\right]$ the molecular diffusion coefficient, $\mathbf{I}$ the identity matrix and $\mathbf{D}\left[\mathrm{L}^{2} \mathrm{~T}^{-1}\right]$ the dispersion tensor characterized by $\alpha_{l}[\mathrm{~L}]$ and $\alpha_{t}[\mathrm{~L}]$, the longitudinal and transverse dispersivities, respectively [Bear, 1972]. Equation (4) is obtained by combining fluid mass balance, eq. (1), and solute mass balance and further applying the Boussinesq approximation. The transport equation (4) is subject to a non-dispersion boundary condition at $z=0$. This implies that the mixing ra- 
tio equals either that of the maximum $(c=1)$ for fluid inflows or that of the resident mass fraction for fluid outflows [Voss and Provost, 2002; Frind, 1982]. For the initial conditions, we consider an horizontal interface between the two fluids located at a specific distance $z_{i}$ from the bottom boundary. Mixing and spreading are quantified from the gradient $g_{z}(x, z, t)=-\partial c(x, z, t) / \partial z$ of the mixing ratio.
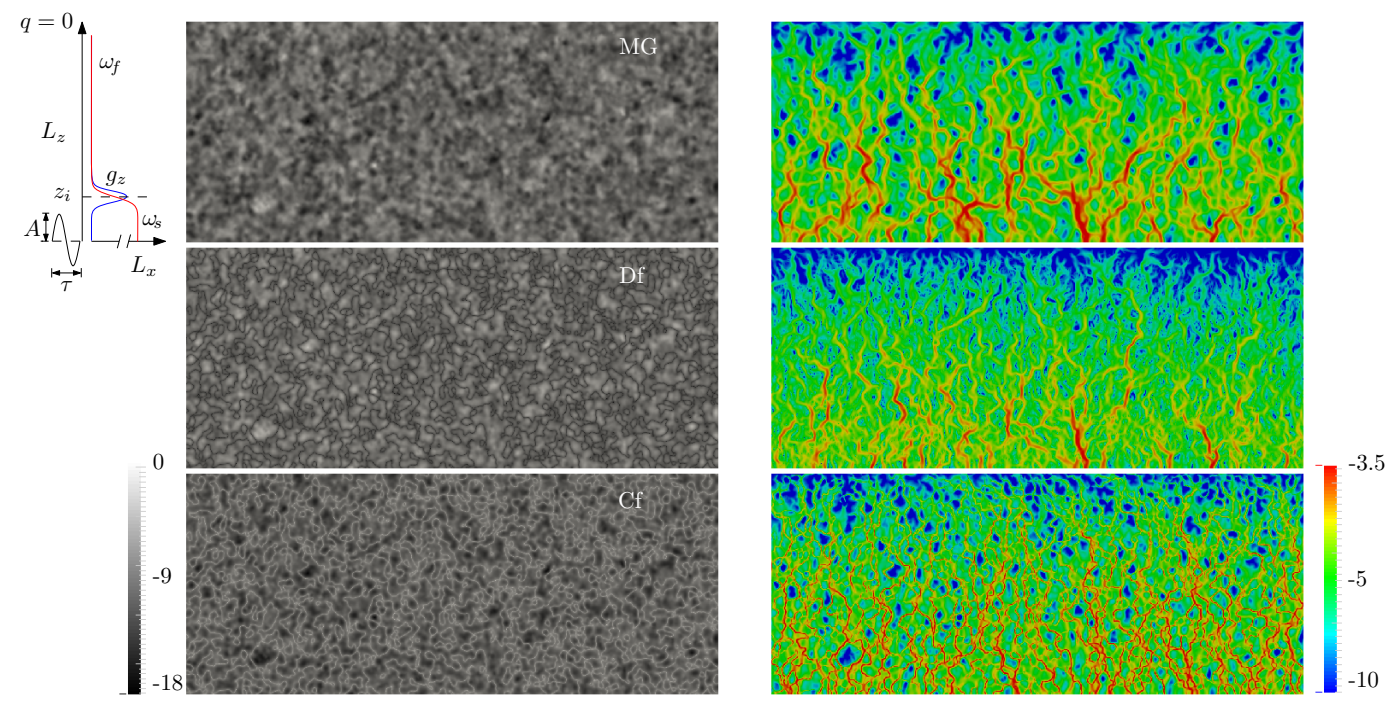

Figure 1. Example of permeability heterogeneous realization $\left(\sigma_{\ln k}^{2}=4\right)$ for the multi-Gaussian (MG), connected (CF) and disconnected (DF) fields and boundary conditions (left) and spatial distribution of the modulus of the velocity $(\mathrm{m} / \mathrm{s})$ for $t=\pi / 2$ (rigth).

This setup may be compared to steady displacement of a solute front [Gramling et al., 2002], or displacement of an initial line source [Le Borgne et al., 2010, 2015]. Note, however, that a steady state flow scenario equivalent to the transient one under consideration here is characterized by 0 flow velocity. An equivalent effective steady state transport model is characterized by a transport velocity that decreases exponentially with distance and an effective dispersion coefficient [Pool et al., 2016]. Thus, temporal fluctuations of the flow boundary conditions are the drivers of the heterogeneity-induced dispersion, mixing and reaction phenomena studied in the following. In the following subsection the reactive transport problem is formulated and the expressions for the reaction rates under mixing-limited conditions are given. Subsection 2.2 defines the measures to characterize solute dispersion and mixing as well as flow deformation, which is are key processes for 
the chemical reactions under consideration. Subsection 2.3 outlines the numerical methodology.

\subsection{Chemical reaction and reaction rate}

In this study, the geochemical setup of calcite dissolution/precipitation, which is very common in variable-density flow systems such as coastal aquifers [see, e.g. Sanford and Konikow, 1989; Rezaei et al., 2005], is considered. The complete reaction system is described in De Simoni et al. [2007]. This system can be characterized by two end-members, freshwater and saline water both under chemical equilibrium conditions with calcite, and its mixing ratio $(c)$. The reaction is assumed to be fast, this means equilibrium is attained instantaneously. Within the reaction system, we focus on the rate of calcite dissolution and precipitation. Note that we do not consider porosity and permeability changes in response to dissolution or precipitation of calcite. Thus, no feedback between the chemical reaction and the flow and transport properties is considered.

We follow the method proposed by De Simoni et al. [2005, 2007], which expresses the equilibrium reaction rate in terms of the mixing ratio $c$ between fresh and saline water as

$$
r_{l}(x, z, t)=\varphi_{w} \rho \frac{\partial^{2} c_{A}}{\partial c^{2}} \nabla c(x, z, t) \cdot\left[\mathbf{D}_{\mathbf{h}}(x, z, t) \nabla c(x, z, t)\right]
$$

where $\varphi_{w}$ is the mole fraction of water and $c_{A}$ the concentration of secondary species [De Simoni et al., 2005]. In the freshwater-saline water mixing problem considered, $c_{A}$ is the molality of $\mathrm{Ca}^{2+}\left(\mathrm{mol} / \mathrm{kg}_{\text {water }}\right)$ [De Simoni et al., 2007]. The reaction rate depends on chemistry through the speciation term $\partial^{2} c_{A} / \partial c^{2}$, and on mixing as expressed by the second term, which is identical to the mixing rate, as discussed below. It is controlled by the gradient of the mixing ratio and the hydrodynamic diffusion-dispersion tensor. The speciation term is determined by using PHREEQC [Parkhurst, 1995] for the end-member compositions given in Table 1 (fresh and saline water). The global reaction rate (moles that precipitate/dissolve in order to keep equilibrium conditions) is defined by areal integration of the local reaction rate, ensemble average over all medium realizations and temporal average over a period of the boundary fluctuations 


$$
r_{g}(t)=\left\langle\frac{1}{\tau} \int_{0}^{\tau} d t^{\prime} \int_{\Omega} d x d z r_{l}\left(x, z, t+t^{\prime}\right)\right\rangle
$$

with $\Omega$ the aquifer domain; the angular brackets denote the ensemble average. The variability of the reaction rate within the domain is characterized in terms of the probability density function $p_{r_{l}}(r)$ of the local reaction rate, which is obtained by spatial sampling of $r_{l}(x, z, t)$ in individual realizations and subsequent ensemble averaging of the resulting single realization PDFs.

Table 1. Chemical composition of the end-members used in the calculations

\begin{tabular}{lccccccc}
\hline Solution & $\mathrm{pH}$ & $\mathrm{Ca}$ & $\mathrm{Mg}$ & $\mathrm{Na}$ & $\mathrm{K}$ & $\mathrm{Cl}$ & $\log P_{\mathrm{CO} 2}$ \\
\hline Saline groundwater & 7.21 & 9.64 & 22.43 & 496.53 & 9.28 & 564.13 & -2.01 \\
Freshwater & 7.30 & 1.65 & 0.00 & 0.00 & 0.00 & 0.00 & -2.00 \\
\hline
\end{tabular}

* Units for concentrations are in $\mathrm{mmol} \mathrm{kg}_{\text {water }}^{-1}$

\subsection{Dispersion and Mixing}

We probe the impact of heterogeneity and density effects on the mixing and spreading dynamics. Dispersion is characterized by the width of the mixing zone between the two fluids, which is quantified here by the second-centered moment of the vertical component of the gradient of the mixing ratio $c$. Mixing is characterized by the mixing rate [see, e.g., Kapoor and Kitanidis, 1998; Fiori and Dagan, 2002; Le Borgne et al., 2010; Bolster et al., 2011] and plays a key role for the dissolution/precipitation reaction under consideration as outlined in the previous section. The local mixing dynamics are related qualitatively to flow deformation and flow topology in terms of the deformation rate tensor. These quantities are defined in the following.

\subsubsection{Interface width}

The interface width characterizes the area between the two fluids and delineates the region of potentially high mixing and reactivity, depending on the local dispersion coefficient and flow deformation, as discussed in the following subsections. The interface width is quantified from the first and second moments of the gradient of the mixing ratio distribution, and reads as 


$$
\sigma_{z}^{2}(x, t)=\left\{m_{z}^{(2)}(x, t)-m_{z}^{(1)}(x, t) m_{z}^{(1)}(x, t)\right\},
$$
tively, are given by

$$
m_{z}^{(i)}(x, t)=\frac{1}{\int g_{z}(z, t \mid x) d z} \int g_{z}(z, t \mid x) z^{i} d z .
$$

Due to the non-uniform flow field induced by the temporal flow fluctuations and the spatial heterogeneity, the second-centered moment of the $z$-component of the gradient of the mixing ratio distribution (7) varies along the $x$-axis. The effective width $\sigma_{e}^{2}(t)$ is given by spatial, ensemble and temporal averaging of $\sigma_{z}^{2}(x, t)$ as

$$
\sigma_{e}^{2}(t)=\left\langle\frac{1}{\tau L_{x}} \int_{0}^{\tau} d t^{\prime} \int_{0}^{L_{x}} d x \sigma_{z}\left(x, t+t^{\prime}\right)\right\rangle .
$$

Note that we perform the temporal average over one period of the boundary fluctuation in order to emphasize the trend.

\subsubsection{Mixing rate}

The mixing rate, or scalar dissipation rate [Tennekes and Lumley, 1972; Pope, 2000] is defined as

$$
\chi_{l}(x, z, t)=\nabla c(x, z, t) \cdot\left[\mathbf{D}_{\mathbf{h}}(x, z, t) \nabla c(x, z, t)\right] .
$$

The reaction rate (5) of the mixing-limited dissolution-precipitation reaction is directly proportional to the mixing rate. The mixing rate quantifies the local mixing mechanisms, namely the existence of concentration gradients and their dissipation by local dispersion. The variability of the mixing rate within the domain is characterized by its $\operatorname{PDF} p_{\chi_{l}}(\chi)$, which is obtained through spatial sampling of $\chi_{l}(x, z, t)$ and subsequent ensemble averaging of the resulting single realization PDFs. The global mixing behavior is measured by the global mixing rate, which is obtained by areal integration, ensemble and temporal average as 


$$
\chi_{g}(t)=\left\langle\frac{1}{\tau} \int_{0}^{\tau} d t^{\prime} \int_{\Omega} d x d z \chi_{l}\left(x, z, t+t^{\prime}\right)\right\rangle .
$$
dient $\gamma_{g}(t)$ is defined in analogy to (11).

For the displacement of a solute front in homogeneous porous medium under constant flow velocity and constant diffusion coefficient $D$, the global mixing rate is $\chi_{g}(t)=$ $\sqrt{D} /(2 \sqrt{2 \pi t})$. Thus, $\chi_{g}$ decays with time due to the diffusive smoothing of the interface between the displacing and the displaced fluids. The square gradient is simply $\gamma_{g}(t)=$ $\chi_{g}(t) / D$. For displacement under unsteady flow conditions in a homogeneous porous medium [Pool et al., 2016], the global mixing rate is

$$
\chi_{g}(t)=\frac{\mathcal{D}(t)+D_{m}}{2 \sqrt{\pi \sigma_{0}^{2}(t)}},
$$

where $\mathcal{D}(t)$ and $\sigma_{0}^{2}(t)$ is given by expressions (36) and (42) in Pool et al. [2016]. As shown there, the gradient of the mixing ratio can be approximated by a Gaussian distribution. Thus, the PDF of local mixing rates $p_{\chi l}(\chi)$ is given by

$$
p_{\chi_{l}}(\chi)=\frac{1}{2 \chi \sqrt{\ln \left(\chi_{m} / \chi_{0}\right) \ln \left(\chi_{m} / \chi\right)}}
$$

where $\chi_{m}=\left(\mathcal{D}+D_{m}\right) /\left(2 \pi \sigma_{0}^{2}\right)$ is the maximum mixing rate at a given time and $\chi_{0}$ a minimum mixing rate. The square gradient $\gamma_{l}$ is related to the mixing rate by rescaling as $\gamma_{l}=\chi_{l} /\left(\mathcal{D}+D_{m}\right)$. Therefore, $\gamma_{l}$ and $\chi_{l}$ differ in their maxima but their spatial distribution is the same.

\subsubsection{Flow Deformation}

The local deformation properties of the flow field are directly related to the mixing and therefore reaction behavior [de Barros et al., 2012; Le Borgne et al., 2013, 2014, 2015; 
Bandopadhyay et al., 2017]. Stretching and compression of the local fluid support leads to a steepening of concentration gradients, which in the presence of local dispersion give rise to enhanced mixing and thus reaction. The deformation of the flow field is characterized by the deformation rate tensor [Okubo, 1970a; de Barros et al., 2012], which describes the local strain and rotation properties of the flow field. It is defined by [Ottino, 1989]

$$
\boldsymbol{\epsilon}(x, z, t)=\left(\begin{array}{ll}
\frac{\partial v_{x}(x, z, t)}{\partial x} & \frac{\partial v_{x}(x, z, t)}{\partial z} \\
\frac{\partial v_{z}(x, z, t)}{\partial x} & \frac{\partial v_{z}(x, z, t)}{\partial z}
\end{array}\right)
$$

with $\mathbf{v}(x, z, t)=\mathbf{q}(x, z, t) / \phi$ the pore water velocity. The vorticity is defined by $\omega=\epsilon_{21}-\epsilon_{12}$, the normal strain and shear rates are defined by $\alpha=2 \epsilon_{11}$ and $\sigma=\epsilon_{21}+\epsilon_{12}$, respectively. The Okubo-Weiss parameter [Okubo, 1970b; Weiss, 1991] is defined in terms of the negative determinant of the deformation gradient tensor $\Theta(x, z, t)=-4 \operatorname{det}[\epsilon(x, z, t)]$. It can be decomposed into $\Theta=\Theta_{S}^{2}-\omega^{2}$, where $\Theta_{\varsigma}=\alpha^{2}+\sigma^{2}$ is a measure for local stretching. Thus, positive values of $\Theta$ are associated with local stretching deformation, negative values with rotation of a fluid element. A spatial map of $\Theta(x, z, t)$ delineates regions of predominant stretching or rotation action of the flow field [Okubo, 1970b; Weiss, 1991]. Regions characterized by strong stretching action can be delineated by $\Theta_{S}(x, z, t)$, which is termed rate of strain in the following. Vorticity has only a minor impact on mixing and mass transfer because it merely describes the rotation of a material element [de Barros et al., 2012]. Strain deformation on the other hand affects the mixing and mass transfer properties through stretching or compression of material elements and subsequent steepending or attenuation of concentration gradients. As the interface passes through a region of high $\Theta_{\varsigma}$, it gets strongly stretched, which steepens the gradient of the mixing ratio [de Barros et al., 2012]. If local scale dispersion is sufficiently strong, stretching leads to enhanced mixing as expressed by the mixing rate (10).

\subsection{Numerical Methodology}

The impact of heterogeneity on mixing and chemical reactions is investigated by performing several sets of Monte Carlo simulations. Spatial heterogeneity in the hydraulic conductivity is characterized by multi-Gaussian lognormally distributed random fields. Medium realizations are generated by using the GCOSIM3D code [Gomez-Hernandez and Journel, 1992]. The geometric mean of hydraulic conductivity $\left(k_{g}\right)$ for all the simulations 
is set to $1.15 \cdot 10^{-4} \mathrm{~m} / \mathrm{s}$. Two different values for the log-conductivity variance are considered $\left(\sigma_{\ln k}^{2}=1\right.$ and 4$)$ and directional correlation in the two spatial dimensions is assumed to be isotropic with correlation length $\lambda=10 \mathrm{~m}$. A model domain of extent $L_{x} \times L_{z}$ is considered, with $L_{x}=10^{2} \lambda$ and $L_{z}=40 \lambda$, discretized in grid cells of size $d_{x} \times d_{z}$, with $d_{x}=\lambda / 10$ and $d_{z}=\lambda / 10$. Moreover, in order to study the effect of channelling patterns, commonly found in sedimentary systems, on mixing and chemical reactions more complex heterogeneous media are considered by following the transformation of the multi-Gaussian fields proposed by Zinn and Harvey [2003]. From this transformation [see also Knudby and Carrera, 2005], we obtain heterogeneous fields characterized by low-conductivity paths (Df, disconnected fields) or high-conductivity paths (Cf, connected fields), see Figure 1. As a criterion for the size of the numerical ensembles, we have chosen the stability of the average of the spatial variance of the front width. The minimum number of realizations to achieve this turned out to be 30 for the multi-Gaussian fields and 40 for the connected fields. The effective values of the previously described observables are computed by performing the average over these realizations.

Furthermore, in order to examine the effect of variable fluid density on mixing and reactions, both constant-density and variable-density saturated flow and transport simulations were performed. Coupled flow and transport equations are solved by using a 2ndorder finite volume and 6th-order compact finite differences code [see details in Hidalgo et al., 2013]. Values for $\alpha_{l}$ and $\alpha_{t}$ of $2 \mathrm{~m}$ and $0.2 \mathrm{~m}$ are considered, which are consistent with those estimated for real systems of this scale [see, e.g. Gelhar et al., 1992; Zech et al., 2015]. Time is scaled with the characteristic advection time defined by $\tau_{a}=A / v_{z}$ with $v_{z}$ the maximum velocity at the boundary for an equivalent homogeneous system considering $k_{g}$ given by $v_{z}=\sqrt{2} A k_{g} \mu / \phi$ [see, e.g., Jacob, 1950; Ferris, 1951], where $\mu=\sqrt{S_{f} \pi /\left(k_{g} \tau\right)}$ represents the inverse of the characteristic distance for the attenuation of the temporal head fluctuations in the domain. In other words, $\gg \mu^{-1}$, denotes the range of the periodic head fluctuations.

Table 2 provides the employed values for the flow and transport simulations and the statistical properties of the heterogeneous fields. Figure 1 shows the model geometry, boundary conditions and (left) the hydraulic conductivity distribution for one random heterogeneous multi-Gaussian realization (MG) and the corresponding transformed disconnected (Df) and connected (Cf) fields after the procedure used by Zinn and Harvey [2003] 


\begin{tabular}{|c|c|c|}
\hline Parameter & Value & Description \\
\hline$L_{x}[\mathrm{~m}]$ & 1000 & Domain $x$ length \\
\hline$L_{z}[\mathrm{~m}]$ & 400 & Domain x length \\
\hline$z_{i}[\mathrm{~m}]$ & 100 & Interface location \\
\hline$k_{g}[\mathrm{~m} / \mathrm{s}]$ & $1.15 \cdot 10^{-4}$ & geometric mean permeability \\
\hline$S_{f}\left[\mathrm{~m}^{-1}\right]$ & $3 \cdot 10^{-5}$ & Specific storage coefficient \\
\hline$\sigma_{\ln k}^{2}$ & 1,4 & variance of the log-hydraulic conductivity \\
\hline$\lambda[\mathrm{m}]$ & 10 & Correlation length \\
\hline$\phi[-]$ & 0.25 & Porosity \\
\hline$\alpha_{L}[\mathrm{~m}]$ & 2 & Longitudinal dispersivity \\
\hline$\alpha_{T}[\mathrm{~m}]$ & 0.2 & Transverse dispersivity \\
\hline$D_{m}\left[\mathrm{~m}^{2} / \mathrm{s}\right]$ & $1 \cdot 10^{-9}$ & Molecular diffusion coefficient \\
\hline$A[\mathrm{~m}]$ & 1 & Oscillation amplitude \\
\hline$\tau[\mathrm{s}]$ & 43200 & Oscillation period \\
\hline
\end{tabular}
velocity $(\mathrm{m} / \mathrm{s})$ for $t=\pi / 2$.

and Knudby and Carrera [2005] and (right) the spatial distribution of the modulus of the

Table 2. Parameters used in numerical simulations.

\section{Results and Discussion}

In the following, we discuss the impact of spatial heterogeneity and connectivity of hydraulic conductivity, and the role of buoyancy on the dispersion mixing and reaction behaviors. To this end, we first focus on the impact of heterogeneity and connectivity for constant fluid density. The obtained behaviors are then compared to the behaviors in the presence of fluid density variations.

\subsection{Spatial Heterogeneity and Connectivity}

We study the impact of spatial heterogeneity and connectivity on the dispersion of the interface between the displacing and displaced fluids, the mixing of the two fluids, and chemical reactivity. Figure 2 illustrates the conductivity distribution for the different heterogeneity scenarios under consideration as well as the distribution of the mixing ratio 
and magnitude of its gradient, which delineates the interface. We consider global behavior of dispersion, mixing and reaction measures defined in Section 2. The spatial variability of mixing and reaction is quantified by the PDFs of the local mixing and reaction rates. The occurrence of mixing and reaction patterns is studied by spatial maps of the local reaction and mixing rates and related to the medium and flow structure through the map of local strain rates.
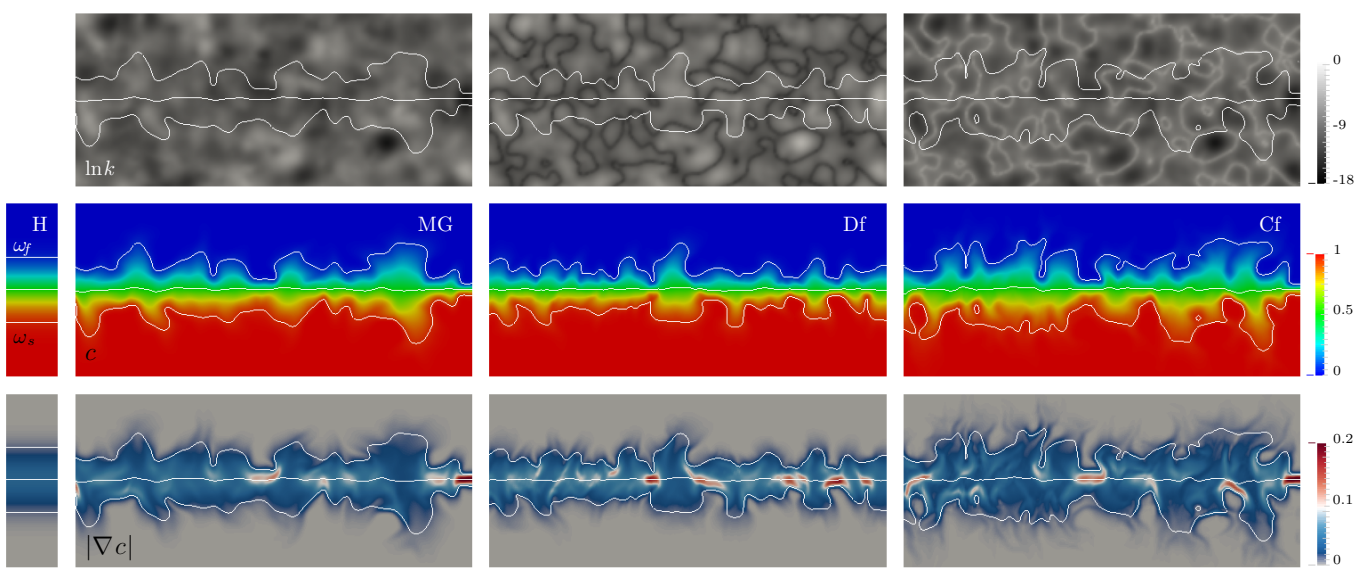

Figure 2. Example of a zoomed-in portion of the hydraulic conductivity distribution $(\ln k)$, snapshot of the mixing ratio distribution $(c)$ and its gradient for the homogeneous case $(\mathrm{H})$ and one heterogeneous multiGaussian (MG), disconnected (Df) and connected (Cf) realization $\left(\sigma_{\ln k}^{2}=4\right)$ at time $t^{\prime}=t / \tau_{a}=74$.

\subsubsection{Dispersion}

Periodic temporal forcing leads to a noninstantaneous flow field, i.e. space and timedependent flow response, in which the oscillation amplitude decays exponentially with distance from the boundary whereas the phase lag increases linearly [see, e.g., Jacob, 1950; Van Der Kamp, 1972; Townley, 1995]. The resulting time-dependent velocity field enhances solute dispersion even if the medium is homogeneous [see, e.g. Kinzelbach and Ackerer, 1986; Goode and Konikow, 1990; Pool et al., 2016].

Figure 3 shows the temporal evolution of the second-centered moment $\left(\sigma_{e}^{2}(t)\right)$ of the gradient of the mixing ratio for different values of the log-conductivity variance $\left(\sigma_{\ln k}^{2}\right)$ and different degrees of connectivity. Results for the equivalent homogeneous case [Pool et al., 2016], which is characterized by the same dispersivities, storativity and porosity as the heterogeneous model and with a unique hydraulic conductivity equal to $k_{g}$, are in- 


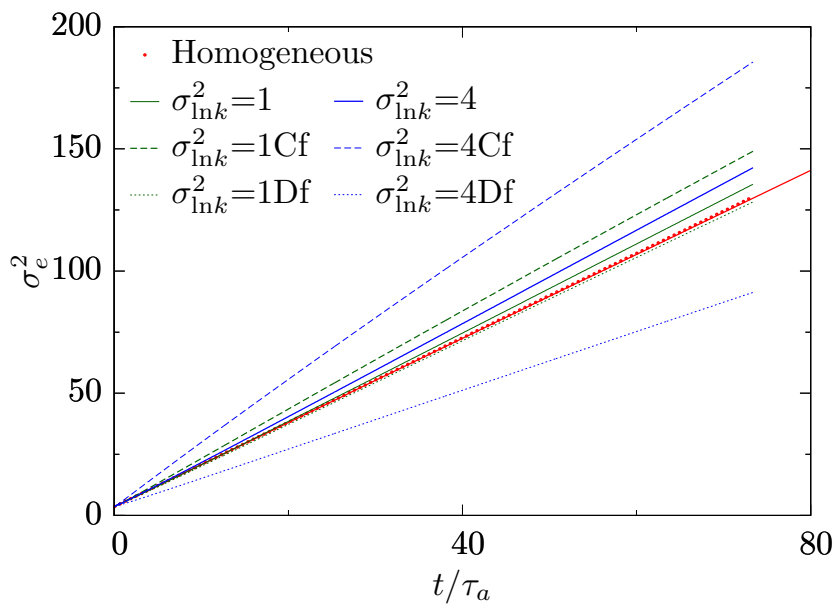

Figure 3. Temporal behavior of $\sigma_{e}^{2}(t)$ for different values of $\sigma_{\ln k}^{2}$ for the multi-Gaussian, connected (Cf) and disconnected (Df) fields. The red line represents the results obtained from the analytical solution derived in Pool et al. [2016] for illustration.

cluded for comparison. Numerical results show that, as expected, for the multi-Gaussian fields the second-centered moment of the gradient of the mixing ratio distribution $\sigma_{e}^{2}(t)$ slightly increases with the log-conductivity variance. Temporal oscillations combined with high degree of connectivity substantially widen the mixing zone between the fresh and saline water, an effect that is significantly enhanced for increasing $\sigma_{\ln k}^{2}$. Thus, for moderately heterogeneous connected permeability fields $\left(\sigma_{\ln k}^{2}=1\right), \sigma_{e}^{2}(t)$ is much larger than that for highly heterogeneous multi-Gaussian permeability fields $\left(\sigma_{\ln k}^{2}=4\right)$. On the other hand, for the disconnected fields, $\sigma_{e}^{2}(t)$ is smaller than the one for the equivalent homogeneous case, and decreases when the log-conductivity variance increases. These results may be attributed to the impact of connectivity on the effective conductivity. While for the multiGaussian fields the effective conductivity corresponds to the geometric mean [see, e.g. Matheron, 1967; Dagan, 1989], for the connected fields it is higher than $k_{g}$ and increases with $\sigma_{\ln k}^{2}$ whereas for the disconnected fields it is lower and decreases with $\sigma_{\ln k}^{2}$ [Zinn and Harvey, 2003]. For the disconnected fields, an increase in $\sigma_{\ln k}^{2}$ promotes the emergence of regions of disconnected high velocity embedded in low-velocity channels, which leads to a decrease in the effective conductivity and then in the transient-induced velocity. As a result, the effect of the temporal fluctuations on solute dispersion decreases leading to a smaller width of the mixing zone. However, for the connected fields the increase in $\sigma_{\ln k}^{2}$ increases the effective conductivity and leads to flow fields characterized by a continuous 
network of high velocity paths. The strong velocity variations induced by these channeling patterns and the periodic temporal fluctuations lead to a significant deformation and elongation of the interface in flow direction such that the width of the mixing zone increases.

The deformation of the interface can be quantified in terms of the temporal change of the length of a particular concentration isoline. The interface length is a measure of deformation on one hand and the length of the contact line between the displacing and displaced fluids. Thus, it is both an alternative measure of dispersion and serves for the quantification of the mixing efficiency. Unlike in fluids, however, where line length, or its rate of increase (Lyapunov exponents) is directly used as a measure for mixing, here the local values of dispersion along the interface play a key role in the quantification of mixing as discussed below.

Figure 4 shows the temporal evolution of the $90 \%$ isoconcentration contour line for the multi-Gaussian, connected and disconnected fields for different values of the logconductivity variance. The elongation of this specific isoline evolves as a power law of time with the exponent increasing with the log-conductivity variance [Le Borgne et al., 2013]. Note that for uniform steady flow in heterogeneous media, one would expect an initially linear growth of the interface length caused by persistent flow velocities. For the transient flow scenario under consideration, however, the velocity magnitude decreases in the direction of the flow [Pool et al., 2016], which then leads to a slower increase of the interface length. At distances from the inlet boundary that are smaller than the correlation length, segments of the interface move as in 1-dimensional homogeneous media. The evolution of each segment can be described by the solution for a homogeneous medium as $z_{0}(t)=\ln \left(1+t / \tau_{v}\right) / 2 \mu$, where $\tau_{v}=2 \pi / v^{2} \mu^{2} \tau$, see Eq. (30) in [Pool et al., 2016]. Thus, the initial elongation, the result of persistent velocity contrasts along the interface, i.e., different $v$, grows approximately as $z_{0}(t)$. At later times, velocities decorrelate and the interface evolves sublinearly. Note that, although the deformation of the interface is significantly enhanced by the degree of connectivity and grows faster, similar exponents are obtained for the multi-Gaussian and for the connected fields.

\subsubsection{Mixing rate}

The temporal evolution of the square of the mixing ratio gradient and the global mixing rate obtained by averaging over the period is illustrated in Figure 5. Results reveal 


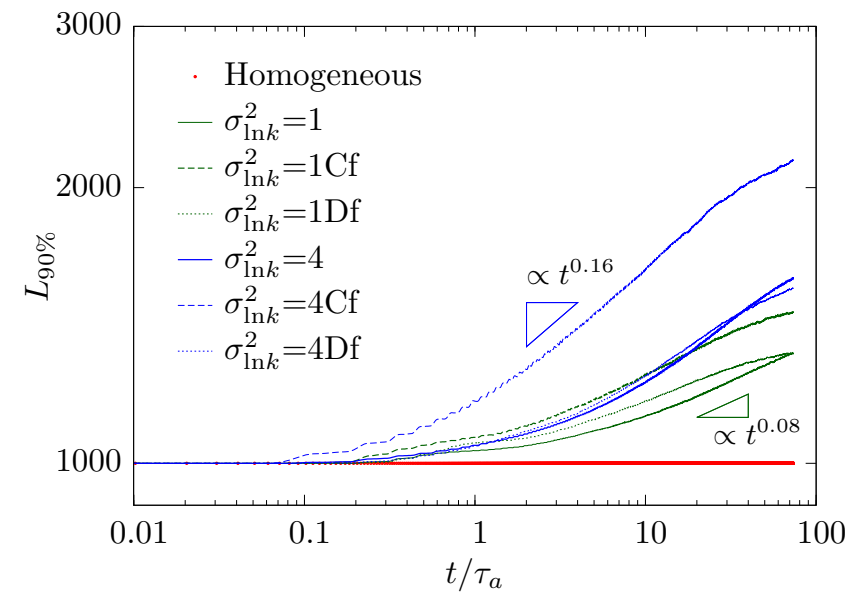

Figure 4. Temporal evolution of the length of the $90 \%$ isoconcentration contour line for the multi-Gaussian, connected (Cf) and disconnected (Df) fields considering different values of the log-conductivity variance.

that heterogeneity significantly impacts on the concentration gradients. Spatial variability of the hydraulic conductivity leads to local velocity variations which promotes segregation and the steepening of concentration gradients [Le Borgne et al., 2013]. This effect, which is magnified by the degree of heterogeneity and connectivity, causes the destruction of the gradients to significantly slow down compared to the homogeneous case, see Figure 5a. For constant dispersion coefficient, this result would suggest a drastic increase in the mixing rate with heterogeneity as it increases with the square of the local concentration gradients. However, for the velocity-dependent dispersion tensor under consideration here, we find that the global mixing rate is (i) generally smaller than the one for the homogeneous scenario, and (ii) decreases for increasing heterogeneity in all cases, see Figure 5b. This behavior is due to the velocity dependence of dispersion. The largest gradients are located at low conductivity zones where velocities and thus dispersion are very small, see Figure 2. Thus, while heterogeneity-induced segregation leads to a strong increase of concentration gradients, it lowers the global mixing efficiency due to the lack of dispersion in the regions of the highest gradients. As shown in Figure 2, concentration gradients are also enhanced in the vicinity of high conductivity zones due to strong velocity variations, which can be clearly seen for the connected fields. As discussed below this increase of the mixing ratio gradients is linked to increased strain deformation. Thus, as local dispersion is significantly enhanced in high conductivity zones this leads to high local mixing rates. However, due to the strong spatial segregation, spatial heterogeneity has less of an impact on the global mixing rate, but may have a significant impact on the spatial distribution and 
variability of local mixing rates, and leads to localization in regions of high mixing rate as discussed below.
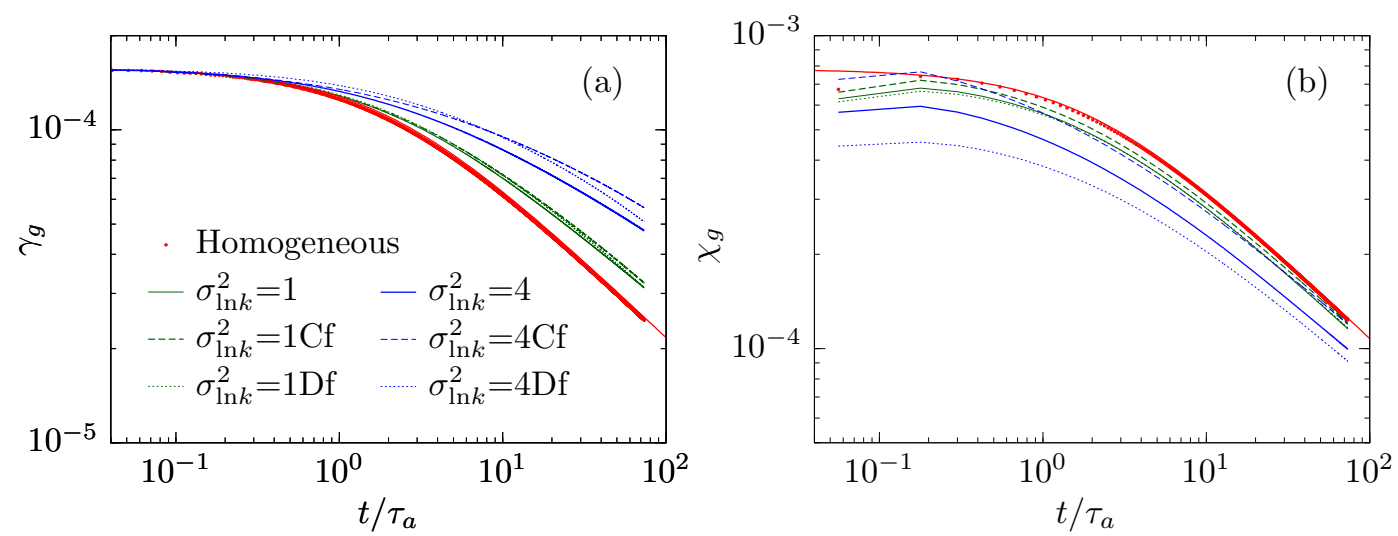

Figure 5. Temporal behavior of the square of the mixing ratio gradient and the mixing rate for the multiGaussian, connected (Cf) and disconnected (Df) fields for different values of the log-conductivity variance.

The spatial variability of the mixing rate induced by structural heterogeneity can be characterized by the PDF $p_{\chi_{l}}(\chi)$ of its local values. As described in Section 2.2.2, the PDF is obtained by spatially sampling all values in the model domain that are above or equal to a specific threshold $\left(\chi_{l} \geq 10^{-6}\right)$. Figure 6 shows $p_{\chi_{l}}(\chi)$ at time $t=74 \tau_{a}$, which coincides with a maximum of the head signal at the boundary. Note that the behaviors of the PDF of the temporally averaged reaction rates (not shown) are qualitatively similar to ones at the maximum time. Also, at this time the global mixing rates for the connected and homogeneous scenarios have similar values (see Figure 5b). The obtained distribution of local mixing rates for the heterogeneous realizations, however, is much broader for the heterogeneous than the for the homogeneous case, which reflects the spatial heterogeneity in flow velocity and dispersion. The PDF for the homogeneous case, Eq. (14), behaves as $\chi^{-1}$ at small values of the mixing rate, which is characteristic of a Gaussian shaped spatial distribution. At higher values there is a cut-off at the maximum concentration, where $p_{\chi l}(\chi)$ has an integrable singularity. The PDFs for the heterogeneous cases behave as $\chi^{-1}$ at small mixing rates, which indicate a quasi-Gaussian behavior of the spatial distribution of $\chi_{l}$ at least for small values of $\chi_{l}$. However, the range of $\chi$ is much larger for the heterogeneous scenarios and the behavior at large $\chi$ is very different. Instead of a singular behavior like in the homogeneous case, $p_{\chi_{l}}(\chi)$ decreases smoothly to zero. This type of behavior can be explained by a broad distribution of local maxima of the mixing rate 
as illustrated in Figure 9. Thus, the PDF of mixing ratios $p_{\chi l}(\chi)$ may be understood as a superposition of local PDFs corresponding to (14) for different (local) maximum mixing ratios $\chi_{m}$. This argument will be explored in future work. Note that as the degree of connectivity increases, the occurrence of high values of the mixing ratio increases. This increase, which reflects high dispersive mass transfer processes, demonstrates that spatial variability of hydraulic conductivity and especially channelling patterns lead to locally enhanced mixing. Therefore, while the global behavior of the mixing rate is not informative on the mixing behavior in the different heterogeneity scenarios, the PDF of local mixing ratios, which play a key role in the effective reaction efficiency, reflects the local mixing mechanisms which are controlled by the local spatial structure of the velocity field. The global mixing rate for the heterogeneous cases may be smaller than the one for the homogeneous case due to segregation of the spatial distribution of the mixing ratio, but the occurrence of high local values increases, which is an indicator for the creation of mixing and reaction hotspots as discussed below.

\subsubsection{Reaction rate}

In order to quantify the effect of heterogeneity and connectivity on chemical reactions, we first consider the global reaction rate given by equation (6). In order to compute the local reaction rates, the second derivative of $c_{A}$ with respect to the mixing ratio $c$ is evaluated as described in Section 2.1.

The behavior of the reaction rate for the different heterogeneity scenarios is very similar to those observed for the mixing rate in the previous section, which is expected due to the strong relation between mixing and reaction rates expressed in Eq. (6). Figure 7 shows the temporal evolution of the global reaction rate for different values of $\sigma_{\ln k}^{2}$ and different degrees of connectivity. Similar to the global mixing rate, Figure 5b, numerical results show that the global reaction efficiency is higher for the homogeneous than those for all of the heterogeneous scenarios. Moreover, the global reaction efficiency decreases with increasing $\sigma_{\ln k}^{2}$, which again can be traced back to the increase of segregation and the lack of dispersion in low conductivity and thus velocity regions. As for the mixing rate, this segregation on one hand leads to a reduction of the global reaction rate, on the other it leads to a broadening of the distribution of local reaction rates with localization of of high reaction rates in regions of high dispersive mass transfer. 

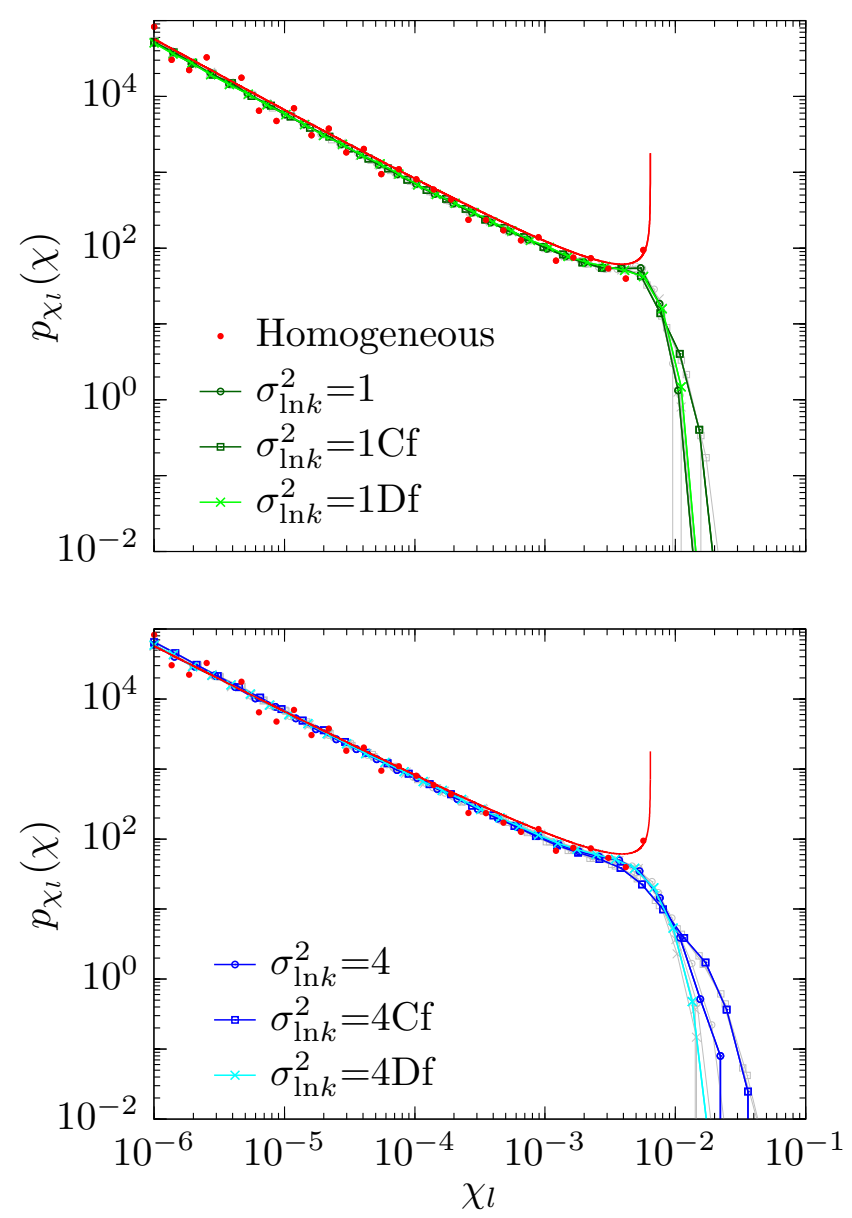

Figure 6. PDF of the local mixing rate for the multi-Gaussian, connected (Cf) and disconnected (Df) fields considering different values of the log-conductivity variance at time $t^{\prime}=t / \tau_{a}=74$, which corresponds to the maximum of the head signal at the boundary. The grey symbols denote corresponding PDFs in individual realizations, which differ only slightly from the ensemble PDF. The red solid line represents predictions from the equation (14).

The PDF $p_{r_{l}}(r)$ provides a measure for the variability of local reaction rates $r_{l}(x, z, t)$. Figure 8 shows $p_{r_{l}}(r)$ computed at time $t=74 \tau_{a}$ for the multi-Gaussian, connected and disconnected fields for different values of $\sigma_{\ln k}^{2}$. This time has been chosen because it coincides with a maximum of the head signal at the boundary, at which the impact of fluctuations is maximum. The behaviors of the PDF of the temporally averaged reaction rates are qualitatively similar to ones at the maximum time. The results suggest that, although at this specific time the global reaction rate for the homogeneous case is found to be similar as those for the connected scenarios, see Figure 7, the range of local reaction rates is significantly enhanced by the degree of heterogeneity and connectivity. The behavior 


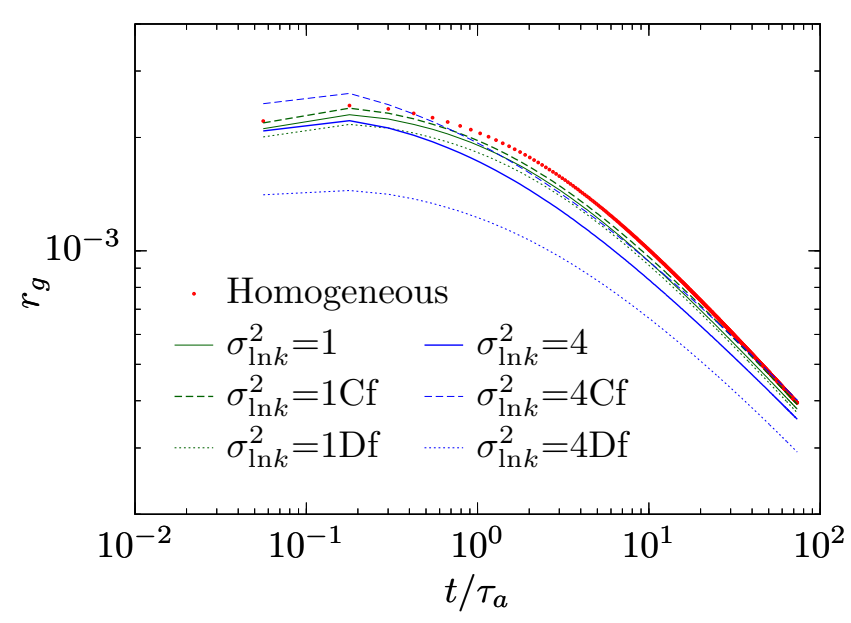

Figure 7. Temporal behavior of the global reactivity for the multi-Gaussian, connected (Cf) and disconnected (Df) fields considering different of the log-conductivity variance.

is similar to the one observed for the PDF of the mixing rates in the previous section. At small reaction rates, we observe a similar $1 / r$ decay that can be attributed to a Gaussian shape of the spatial distribution of $\chi_{l}$ and thus of $r_{l}$. The behavior at large $r$ is again due to a broad distribution of maximum reaction rates in the heterogeneous medium. The maximum reactions rates for all the heterogeneous realizations are significantly higher than those for the homogeneous case, up to over one order of magnitude larger for the strongly heterogeneous connected fields. Therefore, the numerical results demonstrate that the effects of heterogeneity and connectivity on mixing lead to a strongly heterogeneous distribution of local mixing rates, which tends to increase locally the potential mixing and as a consequence reactivity of the system. In the following, we consider the spatial organization of the local mixing and reaction rates.

\subsubsection{Mixing, reaction and deformation patterns}

In order to identify and delineate regions of locally increased mixing reaction potential, we analyze and quantify the deformation of the flow field as described in Section 2.2.3 in terms of the rate of strain $\Theta_{S}(x, z, t)$. The strain rate is expected to increase in areas of high velocity, which together with enhanced dispersion leads to higher mixing and thus reaction rates.

Figure 9 displays a zoomed-in example of, from top to bottom, the hydraulic conductivity and mixing ratio distributions, the strain rate $\left(\Theta_{\varsigma}\right)$, and the local scalar dissipa- 

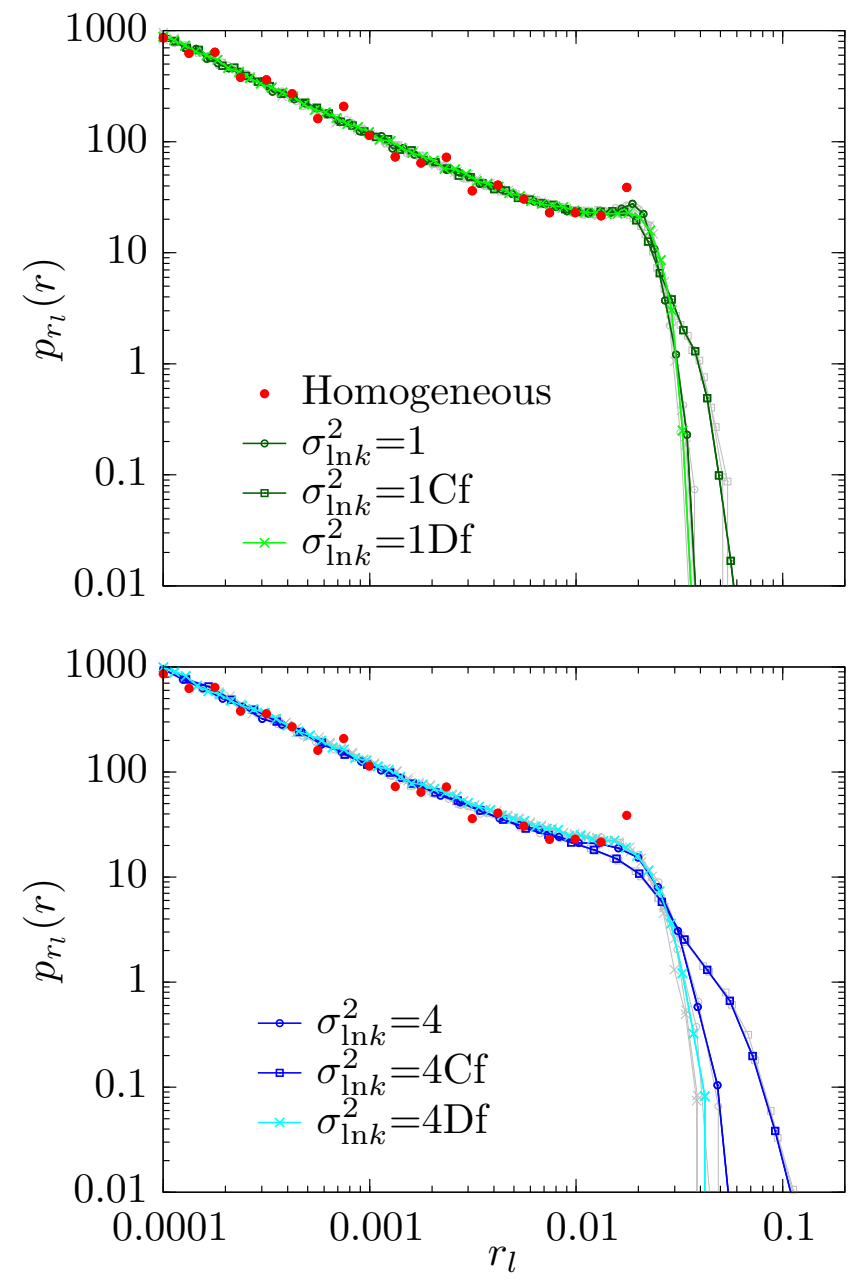

Figure 8. Reaction rate probability density function (PDF) for the multi-Gaussian, connected (Cf) and disconnected (Df) fields considering different of the log-conductivity variance at time $t^{\prime}=t / \tau_{a}=74$, which corresponds to the maximum of the head signal at the boundary. The grey symbols denote corresponding PDFs in individual realizations, which differ only slightly from the ensemble PDF.

tion $\left(\chi_{l}\right)$ and reaction rate distributions $\left(r_{l}\right)$ for the homogeneous case and multi-Gaussian, connected and disconnected fields $\left(\sigma_{\ln k}^{2}=4\right)$ at time $t=74 \tau_{a}$. Note that the strain deformation for the homogeneous field is approximately zero here. This is because, although the storativity is non-zero and then a spatially nonuniform time-dependent velocity field in response to the fluctuations of the lower boundary condition is expected to occur, it is small enough to lead to a practically instantaneous and therefore spatially uniform flow response. Figure 9 reveals that the strain deformation is enhanced by heterogeneity and significantly increases with the degree of connectivity. Connectivity leads to spatial velocity gradients, which strongly promote the emergence of connected patterns of high deformation. This 

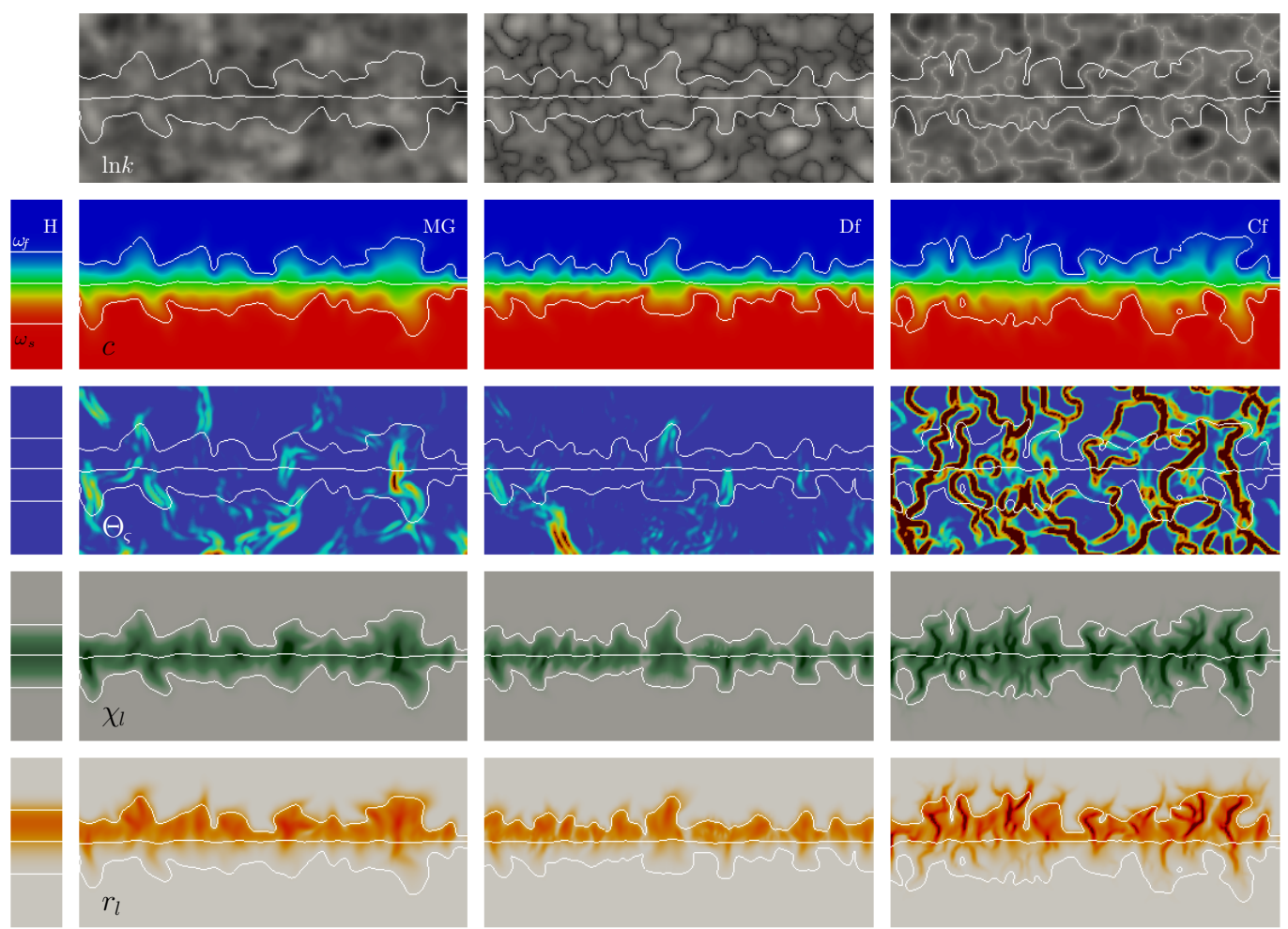

Figure 9. From top to bottom, example of a zoomed-in portion of the $\ln k$ for one conductivity heterogeneous realization for the homogeneous (H), multi-Gaussian (MG), disconnected (Df) and connected (Cf) fields, snapshot of the mixing ratio distribution, local values for the $\Theta_{\varsigma}$ parameter and the local scalar dissipation $\chi_{l}$ and reaction rates $r_{l}$ distribution at time $t^{\prime}=t / \tau_{a}=74$. The white lines represent normalized salinity contours $(5,50$ and $95 \%)$

leads to an elongation of the contact surface between the two fluids, and steepening of mixing ratio gradients in the vicinity of the regions of high conductivity where dispersion is high. As a result, the local mixing and reaction rates are significantly enhanced. Thus, the maximum reaction efficiency and thus dissolution occurs in the regions of highest strain, which are aligned with the connected channels of high conductivity.

Hotspots of geochemical reactivity are clearly related to the deformation of the flow field induced by spatial heterogeneity and the periodic temporal fluctuations. Therefore, evaluation of the deformation properties and topology of the flow field allow to localize and predict regions of enhanced geochemical reaction efficiency. Our results also show that reaction rates are maximal in the freshwater end of the interface [see also Rezaei et al., 2005], particularly between the 5 and 50\% salinity contour lines. This behavior is 
due to the speciation term $\partial^{2} c_{A} / \partial c^{2}$ in Eq. (6) which is maximum for small mixing ratio $(c)$ between the two end-members, and decreases asymptotically with the proportion of saline water [see Figure 5b in De Simoni et al., 2007]. Therefore, a mixing ratio with a small fraction of saline water increases significantly the geochemical reactivity of the system.

Note also that for the homogeneous case a perfectly horizontal reactive front is obtained, whereas the combined effect of spatial variability and temporal fluctuations allow mixing and geochemical reaction patterns to emerge. Thus, irregular reaction conduit networks are obtained, which are characterized by vertical structures, localized where flow velocities are relatively high, and connected to a main horizontal bedding plane.

\subsection{Impact of density variations}

The dispersion of non-reactive tracers in density-dependent groundwater flow systems has been investigated experimentally, theoretically and numerically in a series of studies [see, e.g. Hassanizadeh and Leijnse, 1988; Schotting et al., 1999; Johannsen et al., 2002; Landman et al., 2007a]. These works found that density variations may lead to an increase or a reduction of dispersion, which is controlled by the ratio of viscous to buoyancy forces.
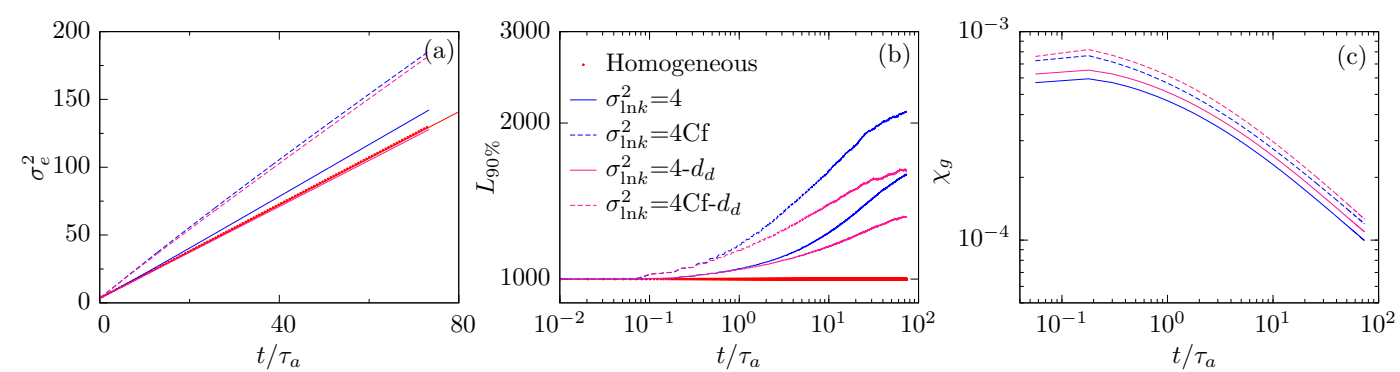

Figure 10. Density-constant and -dependent $\left(d_{d}\right)$ results for the temporal evolution of (a) the secondcentered moment of the mixing ratio gradient in $z$-direction, (b) the length of the $90 \%$ isoconcentration contour line and (c) the scalar disipation rate for the homogeneous, multi-Gaussian (MG) and connected (Cf) fields $\left(\sigma_{\ln k}^{2}=4\right)$.

The impact of density variations on the temporal evolutions of $\sigma_{e}^{2}(t)$, the interface length, and the global mixing rate $\chi_{g}(t)$ for the multi-Gaussian and connected fields is illustrated in Figure 10. Although the observed behaviors are qualitatively similar to those 
obtained for constant density, the data reveal the impact of buoyancy forces on the dispersion and mixing dynamics. For the strongly heterogeneous multi-Gaussian fields $\left(\sigma_{\ln k}^{2}=4\right)$ the variance is dramatically reduced compared to the scenario with constant density, and follows a similar trend as the one obtained for the equivalent homogeneous case. For the connected fields, the variance is slightly reduced in the presence of density variations. The decrease of the width of the mixing zone with density variations is due to the competition of heterogeneity-induced velocity variations (viscous forces) and buoyancy forces. Local density gradients promote downward rotations in the flow field, which in turn lead to a decrease in the emergence of vertical elongations in the interface. As illustrated in Figure 11 , the amplitude of the induced vertical interface elongations, particularly in the freshwater end of the interface, is reduced by buoyancy effects. As a consequence, the deformation of the front and the width of the mixing zone decrease, see Figure 10(a,b).
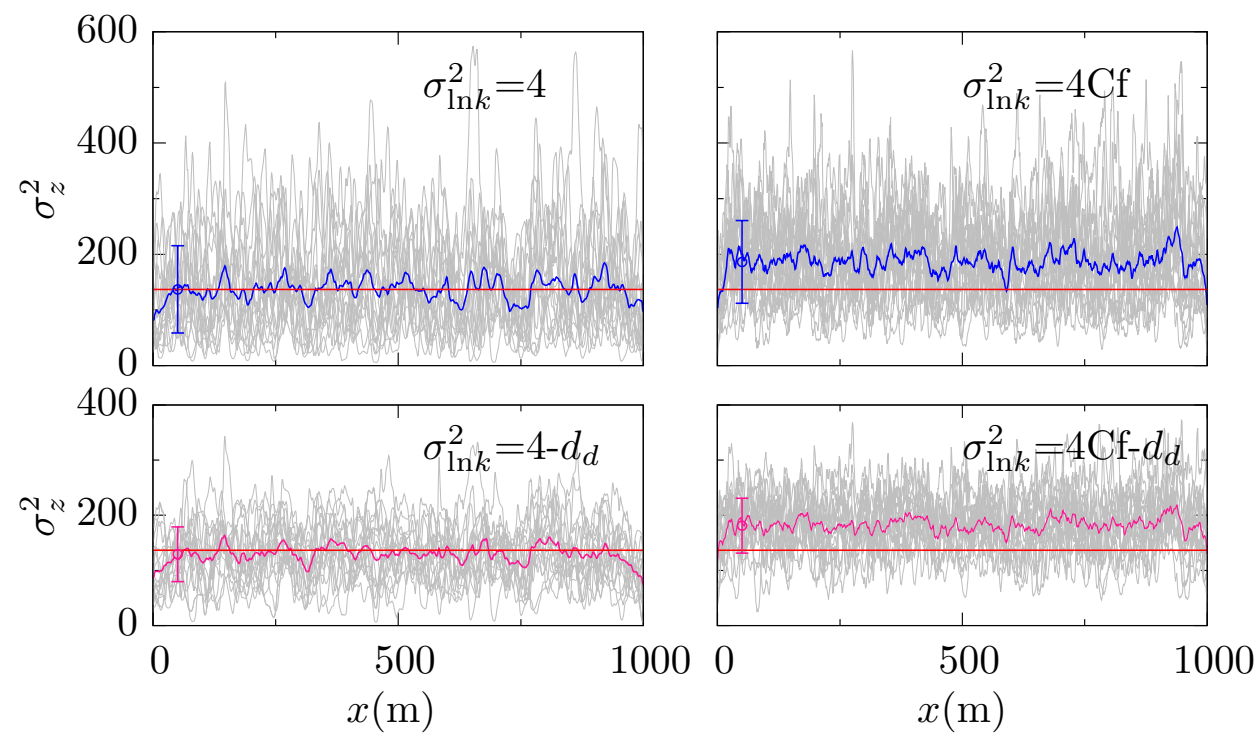

Figure 11. Spatial distribution of the variance of the gradient of the mixing ratio distribution, $\sigma_{z}(x, t)$ equation (7), for the equivalent homogeneous case, red line, and all heterogeneous realizations, gray lines, and ensemble results considering density-constant and density-dependent $\left(d_{d}\right)$ flow, blue and pink lines, respectively, for the multi-Gaussian and connected fields at time $t^{\prime}=t / \tau_{a}=74$.

Thus one may conclude that the mixing rate also decreases due to buoyancy. However, the contrary is the case. The global mixing rate increases with density variations as shown in Figure 10(c). This can be traced back to enhanced compression at the interface. Buoyancy forces lead to a straightening out of the interface, which as discussed 
above manifests in the decreases of its elongation and width $\sigma_{e}(t)$. This straightening out leads to a compression of the interface, which in turn increases the gradients of the mixing ratio in regions of high conductivity and thus high dispersion, see Figure 13. As a result, the global mixing rate increases compared to the scenarios of constant density. This at first counter-intuitive behavior stresses the importance of interfacial compression on the mixing and reaction behavior.
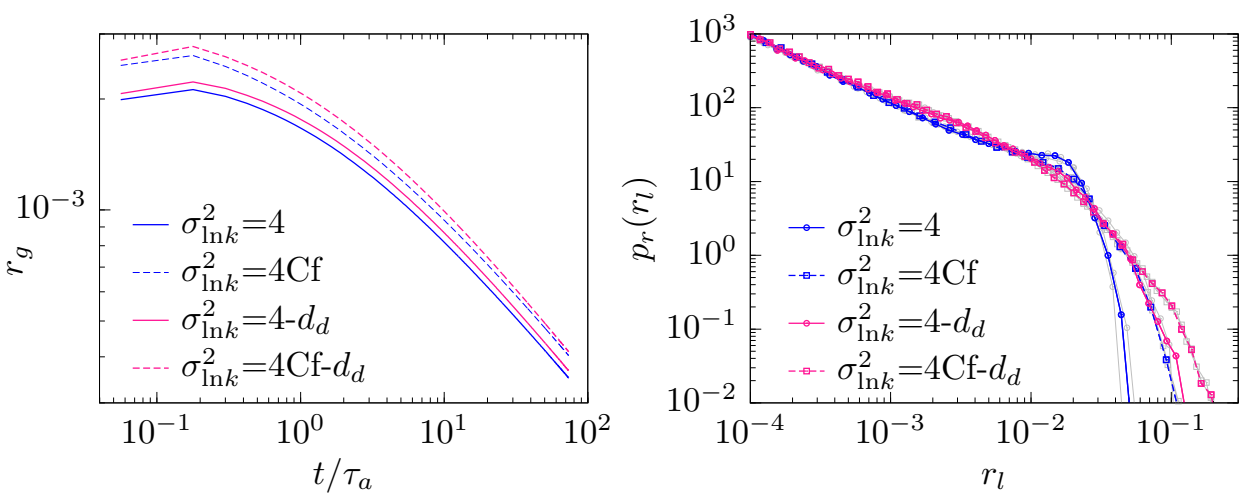

Figure 12. Density-constant and -dependent $\left(d_{d}\right)$ results for the temporal behaviour of the global reactivity (left) and reaction rate probability density function (right) for the multi-Gaussian (MG) and connected (Cf) fields $\left(\sigma_{\ln k}^{2}=4\right)$ at at time $t^{\prime}=t / \tau_{a}=74$, which corresponds to the maximum of the head signal at the boundary. The grey symbols denote corresponding PDFs in individual realizations, which differ only slightly from the ensemble PDF.

In short, buoyancy effects cause concentration gradients to increase in the most permeable regions, which enhances the mixing rate. The increase of the mixing efficiency leads directly to an increase of the reactivity of the system. Figure 12 illustrates the effect of density variations on the evolution of the global reactivity and the PDF of the local reaction rate. Note that, while the global reaction rate slightly increases with density variations compared to the case of constant density, the range of local reaction rates is strongly enhanced by buoyancy effects in both the multi-Gaussian and connected fields. Mixing and reaction are controlled by two primary mechanisms, (i) the combined effect of structural heterogeneity and transient forcing, which leads to enhanced strain in regions of high conductivity and thus dispersion, and (ii) density effects which lead to compaction of the interface, and thus an additional steepening of the concentration gradients. 

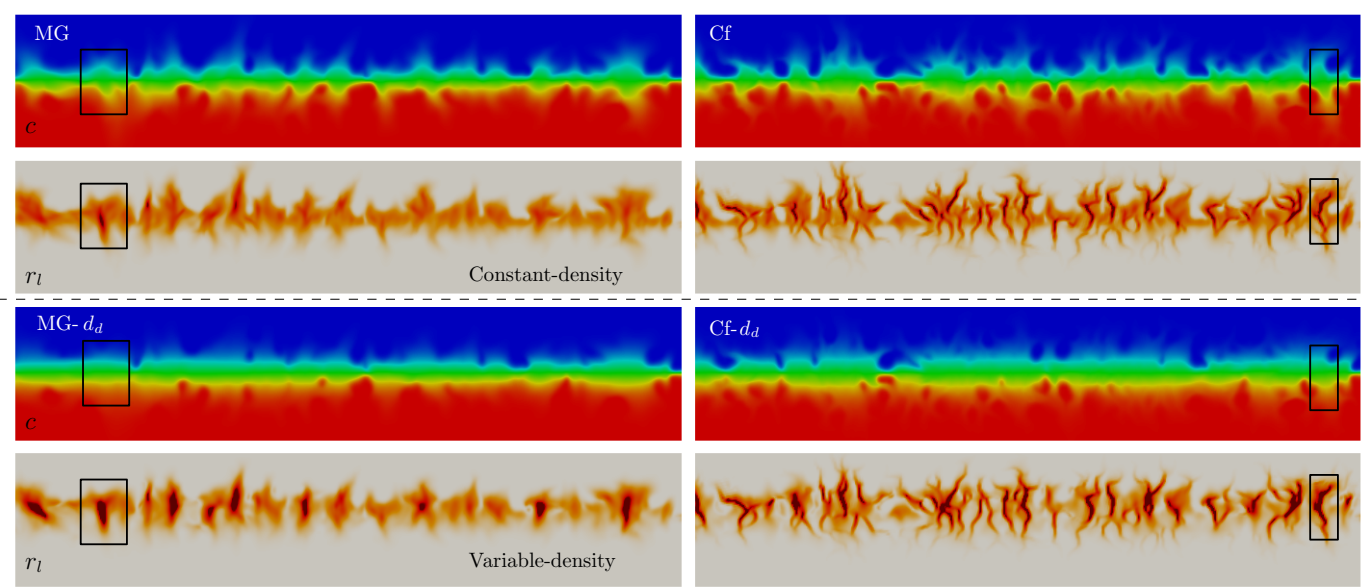

Figure 13. Constant-density and variable-density results for the concentration and reaction rate distribution for one multi-Gaussian field $\left(\sigma_{\ln k}^{2}=4\right)$.

Figure 13 compares the distributions of the mixing ratio and local reaction rates under constant and variable density. The patterns are qualitatively similar. However, as previously discussed, density variations induce an enhancement of the concentration gradients in high-velocity zones leading to a significant increase in the local reaction rates (black squares). This results in a widening of the conduit section. It can be expected that this effect is amplified when accounting for porosity and permeability changes. Moreover, although the deformation of the mixing zone decreases with buoyancy effects and the shape of the interface becomes more horizontal, complex reaction patterns emerge with a vertical development of conduits which end sharply and are connected to a central horizontal structure. This configuration is very common in coastal karst aquifers where there are large main chambers, which can provide a measure of the glacio-eustatic sea level positions [Mylroie and Carew, 1988], with interconnected passages and vertical conduits [see, e.g., Williams, 1977; Mylroie and Carew, 1995; Stoessell et al., 1989; Stoessell, 1995]. Therefore, the coupling of structural heterogeneity, transient forcing and density-driven flow can be a very likely mechanism which would provide maze reaction network patterns where the induced dispersive mass transfers mechanisms would be the dominant control on the configuration of the conduits and cave formation. 


\section{Summary and Conclusions}

We study the interplay between temporal fluctuations, heterogeneity and density effects on mixing and chemical reactions between two fluids of different density under a stable stratification. In a two-dimensional stochastic modeling framework, dispersion and mixing are quantified by the interface width, the global mixing rate and the PDF of local mixing rates. We consider the mixing-induced dissolution of calcite. The reaction rate is determined from a mixing ratio-based formulation which decouples the solute transport and chemical speciation problem [De Simoni et al., 2005].

We find that temporal fluctuations and heterogeneity slightly increase the width of the mixing zone between the two fluids, and that this effect is significantly enhanced by the degree of connectivity. Channeling patterns of high hydraulic conductivity promote significant velocity variations which leads to a strong deformation of the interface between the two fluids. This deformation, characterized by vertical interface elongations, evolves as a power law and stabilizes at asymptotic times. We also find that heterogeneity and transient forcing lead to a spatially nonuniform distribution of dispersion and concentration gradients. Hence, in high conductivity zones dispersive mass transfer is considerably enhanced. On the other hand, in low conductivity zones velocities and dispersion are very low which induces local maxima of the concentration gradients. This significant spatial variability leads to a wide distribution of the local mixing rates, whose maxima increase with the degree of heterogeneity and connectivity. However, this effect is not reflected in the behavior of the global mixing rate as a result of strong segregation, this means the regions of high mixing rate occupy only a small fraction of the domain. This suggests that the global mixing rate is not very informative of the mixing efficiency. Similar behaviors are observed for the global and local reaction rates. While the global reaction rate behaves in a similar way for all the heterogeneous fields, a strong spatial variability is observed for the local reaction rate distribution where the local potential reactivity is strongly increased by heterogeneity and connectivity. The variability in the local mixing and reaction rates is illustrated by their probability density functions, which are obtained through spatial sampling and contrasted to the corresponding PDFs for an equivalent homogeneous medium. We observe a clear increase in the range of mixing and reaction rates towards high values due to heterogeneity, particularly in the connected fields. Note that features such as connectivity depend on the spatial dimensions [Fiori and Jankovic, 2012]. Thus, while the 
basic mechanisms are expected to be qualitatively the same in 3 dimensions, they may be quantitatively different.

Numerical results provide evidence that hotspots of chemical reactivity, this means zones of locally enhanced reaction rates, are clearly related to the deformation of the flow field induced by the coupling of spatial heterogeneity and transient forcing. Thus, hotspots are found to be localized in strongly stretched regions corresponding to high conductivity zones. Therefore, the evaluation of the strain tensor and topology of the flow field allows to delineate reaction patterns and predict regions high reactivity.

When density variations are included, there is a competition between viscous and buoyancy forces. While viscous forces induced by heterogeneity and temporal fluctuations promote the deformation of the interface, density variations tend to stabilize the shape of the mixing zone and favor an horizontal interface. As a result, the width of the mixing zone decreases. This interface compression leads to an increase of the concentration gradients in high conductivity zones which in turn enhanced mixing and reaction rates. Therefore, the interplay between spatial heterogeneity, density-driven flow and transient forcing magnify geochemical processes and the dissolution efficiency. Moreover, our results provide a potential explanation for the formation of complex geochemical patterns observed karst systems, with vertical conduit developments connected to main horizontal bedding caves and chambers. Further analysis considering larger amplitude sea-level fluctuations such as regressions and transgressions or tectonic up- or down-lift displacements is required to explain more complex network patterns observed, for example, in coastal or island karst aquifers. In addition to that, other factors, such as the increase in dimensionality of the media as well as the coupling of changes in permeability due to the increase of porosity, may affect transport and flow patterns and then enhance mixing and reactivity, which should be examined in future investigations.

\section{Acknowledgments}

The data used in this paper can be obtained upon request from the corresponding author. Maria Pool acknowledges the support of the Juan de la Cierva Incorporacion grant (MINECO, Spain). Juan J. Hidalgo is gratefully acknowledged for the valuable assistance in the use of his code. The support of the European Research Council (ERC) through the project MHetScale (617511) is gratefully acknowledged. The authors also acknowledge the support of the Ministry of Economy, Industry and Competitiveness through the projects 
$\mathrm{R})$.

\section{References}

Abarca, E. (2006), Seawater intrusion in complex geological environments, phd thesis, $\mathrm{Ph} . \mathrm{D}$. thesis, Tech. Univ. of Catalonia, Spain.

Appelo, C., and A. Willemsen (1987), Geochemical calculations and observations on salt water intrusions, i. a combined geochemical/minxing cell model, Journal of Hydrology, 94(3), 313-330, doi:http://dx.doi.org/10.1016/0022-1694(87)90058-8.

Appelo, C. A. J. (1994), Cation and proton exchange, ph variations, and carbonate reactions in a freshening aquifer, Water Resour. Res., 30(10), 2793-2805, doi: 10.1029/94WR01048.

Bandopadhyay, A., T. Le Borgne, Y. Meheust, and M. Dentz (2017), Enhanced reaction kinetics and reactive mixing scale dynamics in mixing fronts under under shear flow for arbitrary damkÃúhler numbers, Adv. Water Resour., 100, 78-95, doi: 10.1016/j.advwatres.2016.12.00s.

Bear, J. (1972), Dynamics of Fluids in Porous Media, 764 pp., Elsevier, Amsterdam.

Berkowitz, B., A. Cortis, M. Dentz, and H. Scher (2006), Modeling non-fickian transport in geological formations as a continuous time random walk, Rev. Geophys., 44, RG2003.

Bianchi, M., C. Zheng, C. Wilson, G. Tick, G. Liu, and S. Zheng (2011), Spatial connectivity in a highly heterogeneous aquifer: From cores to preferential flow paths, Water Resour. Res., 47, doi:doi:10.1029/2009WR008966.

Bolster, D., F. ValdÃls-Parada, T. LeBorgne, M. Dentz, and J. Carrera (2011), Mixing in confined stratified aquifers, Journal of Contaminant Hydrology, 120, 198 - 212, doi: http://dx.doi.org/10.1016/j.jconhyd.2010.02.003, reactive Transport in the Subsurface: Mixing, Spreading and Reaction in Heterogeneous Media.

Cirpka, O., and S. Attinger (2003), Effective dispersion in heterogeneous media under random transient flow conditions, Water Resour. Res., 39(9).

Cirpka, O., R. Schwede, J. Luo, and D. M. (2008), Concentration statistics for mixingcontrolled reactive transport in random heterogeneous media, Journal of Contaminant Hydrology, 98(1), 61-74, doi:http://dx.doi.org/10.1016/j.jconhyd.2008.03.005.

Dagan, G. (1987), Theory of solute transport by groundwater, Annu. Rev. Fluid Mech., 19, 183-215. 
Dagan, G. (1989), Flow and transport in porous formations, 465 pp., Springer-Verlag, Berlin Heidelberg New York.

Dagan, G., A. Bellin, and Y. Rubin (1996), Lagrangian analysis of transport in heterogeneous formations under transient flow conditions, Water Resour. Res., 32(4), 891 - 899, doi:10.1029/95wr02497.

de Anna, P., J. Jimenez-Martinez, H. Tabuteau, R. Turuban, T. Le Borgne, M. Derrien, and Y. MÃlheust (2014a), Mixing and reaction kinetics in porous media: An experimental pore scale quantification, Environmental Science \& Technology, 48(1), 508-516, doi:10.1021/es403105b.

de Anna, P., M. Dentz, A. M. Tartakovsky, and T. Le Borgne (2014b), The filamentary structure of mixing fronts and its control on reaction kinetics in porous media flows, Geophys. Res. Lett., 41, doi:10.1002/2014GL060068.

de Barros, F. J., M. Dentz, J. Koch, and W. Nowak (2012), Flow topology and scalar mixing in spatially heterogeneous flow fields, Geophysical Research Letters, 39(8), doi: 10.1029/2012GL051302, 108404.

de Dreuzy, J., J. Carrera, M. Dentz, and T. Le Borgne (2012), Asymptotic dispersion for two-dimensional highly heterogeneous permeability fields under temporally fluctuating flow, Water Resour. Res., 48(1), doi:10.1029/2011WR011129.

De Simoni, M., J. Carrera, X. Sanchez-Vila, and A. Guadagnini (2005), A procedure for the solution of multicomponent reactive transport problems, Water Resources Research, 4l(11), doi:10.1029/2005WR004056, w11410.

De Simoni, M., X. Sanchez-Vila, J. Carrera, and M. W. Saaltink (2007), A mixing ratiosbased formulation for multicomponent reactive transport, Water Resources Research, 43(7), doi:10.1029/2006WR005256, w07419.

Dentz, M., and J. Carrera (2003), Effective dispersion in temporally fluctuating flow through a heterogeneous medium, Phys. Rev. E, 68(3).

Dentz, M., T. Le Borgne, A. Englert, and B. Bijeljic (2011), Mixing, spreading and reaction in heterogeneous media: A brief review., J. Contam. Hydrol., 120, 1-17.

Diersch, H.-J. G., and O. Kolditz (2002), Variable-density flow and transport in porous media: approaches and challenges, Adv. Water Resour., 25(8-12), 899-944.

Ferris, J. (1951), Cyclic fluctuations of water level as a basis for determining aquifer transmissibility, Int. Assoc. Sci. Hydrol., 33, 148-155. 
Fiori, A., and G. Dagan (2002), Transport of a passive scalar in a stratified porous medium, Transport in Porous Media, 47(1), 81 - 98, doi:10.1023/A:1015079408153.

Fiori, A., and I. Jankovic (2012), On preferential flow, channeling and connectivity in heterogeneous porous formations, Math Geosci., 44, 133-145.

Fogg, G., C. Noyes, and S. Carle (1998), Geologically based model of heterogeneous hydraulic conductivity in an alluvial setting, Hydrogeology Journal, 6(1), 131-143, doi: $10.1007 / \mathrm{s} 100400050139$.

Fogg, G. E., S. Carle, and C. Green (2000), Connected-network paradigm for the alluvial aquifer system, Geol. Soc. Am. Spec. Pap., 348, 25-42, doi:doi:10.1130/0-8137-23485.25 .

Frind, E. (1982), Simulation of long-term transient density-dependent transport in groundwater, Adv. Water Resour., 5, 73-88.

Gelhar, L. W. (1993), Stochastic subsurface hydrology, Prentice Hall.

Gelhar, L. W., C. Welty, and K. Rehfeldt (1992), A critical review of data on field-scale dispersion in aquifers, Water Resources Res, 28(7), 1955 - 1974, doi: 10.1029/92WR00607.

Giménez-Forcada, E. (2010), Dynamic of sea water interface using hydrochemical facies evolution diagram, Ground Water, 48(2), 212-216, doi:10.1111/j.17456584.2009.00649.x.

Gomez-Hernandez, J., and A. Journel (1992), Joint sequential simulation of multigaussian fields, Geostat Troia, 1, 85-94.

Gomis-Yagües, V., N. Boluda-Botella, and F. Ruiz-Beviá (1997), Column displacement experiments to validate hydrogeochemical models of seawater intrusions, J. Contam. Hydrol, 29(1), 81-91.

Goode, D. J., and L. Konikow (1990), Apparent dispersion in transient groundwater flow, Water Resour. Res., 26(10), 2339-2351.

Gramling, C. M., C. F. Harvey, and L. C. Meigs (2002), Reactive transport in porous media: A comparison of model prediction with laboratory visualization, Environ. Sci. Technol., 36, $2508-2514$.

Hanshaw, B., and W. Back (1979), The major geochemical processes in the evolution of carbonate-aquifer system, J. Hydrol., 43, 287-312.

Hassanizadeh, S., and T. Leijnse (1988), On the modeling of brine transport in porous media, Water Resources Research, 24(3), 321-330, doi:10.1029/WR024i003p00321. 
Held, R., S. Attinger, and W. Kinzelbach (2005), Homogenization and effective parameters for the henry problem in heterogeneous formations, Water Resour. Res., 41, doi: 10.1029/2004WR003674

Hidalgo, J. J., C. W. MacMinn, and R. Juanes (2013), Dynamics of convective dissolution from a migrating current of carbon dioxide, Adv. Water Resour., 62, $511-519$, doi: http://dx.doi.org/10.1016/j.advwatres.2013.06.013, computational Methods in Geologic $\mathrm{CO} 2$ Sequestration.

Hidalgo, J. J., M. Dentz, Y. Cabeza, and J. Carrera (2015), Dissolution patterns and mixing dynamics in unstable reactive flow, Geophys. Res. Lett., 42, 6375-6364, doi: doi:10.1002/2015GL065036.

Izgec, O., D. Birol, B. Henri, and A. Serhat (2008), Co2 injection into saline carbonate aquifer formations i: laboratory investigation, Transport in Porous Media, 72(1), 1-24, doi:10.1007/s11242-007-9132-5.

Jacob, C. E. (1950), Flow of groundwater, in Engineering Hydraulics, H. Rouse, John Wiley, New York, p321-386.

Johannsen, K., W. Kinzelbach, S. Oswald, and G. Wittum (2002), The saltpool benchmark problem - numerical simulation of saltwater upconing in a porous medium, Adv. Water Resour., 25(3), 335-348.

Kapoor, V., and P. K. Kitanidis (1998), Concentration fluctuations and dilution in aquifers, Water Resources Research, 34(5), 1181-1193, doi:10.1029/97WR03608.

Kerrou, J., and P. Renard (2010), A numerical analysis of dimensionality and heterogeneity effects on advective dispersive seawater intrusion processes, Hydrogeol. J., 18, 5572 .

Kinzelbach, W., and P. Ackerer (1986), Mode'lisation de la propagation d'un contaminant dans un champ d'e'coulement transitoire, Hydrogeologie, 2, 197-206.

Knudby, C., and J. Carrera (2005), On the relationship between indicators of geostatistical, flow and transport connectivity, Adv. Water Resour., 28(4), 405-421, doi: http://dx.doi.org/10.1016/j.advwatres.2004.09.001.

Landman, A., R. Schotting, A. Egorov, and D. Demidov (2007a), Densitydependent dispersion in heterogeneous porous media part ii: Comparison with nonlinear models, Adv. Water Resour., 30(12), 2481-2498, doi: http://dx.doi.org/10.1016/j.advwatres.2007.05.017. 
Landman, A. J., K. Johannsen, and R. Schotting (2007b), Density-dependent dispersion in heterogeneous porous media part i: A numerical study, Adv. Water Resour., 30(12), 2467-2480, doi:http://dx.doi.org/10.1016/j.advwatres.2007.05.016.

Le Borgne, T., M. Dentz, D. Bolster, J. Carrera, J.-R. de Dreuzy, and P. Davy (2010), Non-fickian mixing: Temporal evolution of the scalar dissipation rate in heterogeneous porous media, Adv. Water Resour., 33(12), 1468-1475, doi: 10.1016/j.advwatres.2010.08.006.

Le Borgne, T., M. Dentz, and E. Villermaux (2013), Stretching, coalescence, and mixing in porous media., Phys. Rev. Lett., 110(20).

Le Borgne, T., T. Ginn, and M. Dentz (2014), Impact of fluid deformation on mixinginduced chemical reactions in heterogeneous flows, Geophysical Research Letters, 41(22), 7898-7906, doi:10.1002/2014GL062038.

Le Borgne, T., M. Dentz, and E. Villermaux (2015), The lamellar description of mixing in porous media, J. Fluid Mech., 770, 458-498.

Magaritz, M., and J. Luzier (1985), Water-rock interactions and seawater-freshwater mixing effects in the coastal dunes aquifer, coos bay, oregon, Geochimica et Cosmochimica Acta, 49(12), 2515-2525, doi:http://dx.doi.org/10.1016/0016-7037(85)90119-X.

Magaritz, M., L. Goldenberg, U. Kafri, and A. Arad (1980), Dolomite formation in the seawaterâĂŞfreshwater interface, Nature, 287(5783), 622-624.

Matheron, G. (1967), Eleḿents pour une theórie des milieux poruex, Masson, Paris.

Müller, G., and R. Fischbeck (1973), Possible natural mechanism for protodolomite formation, nature physical science, 242, 139-141.

Mylroie, J., and J. Carew (1988), Solution conduits as indicators of late quaternary sea level position, Quaternary Science Reviews, 7(1), 55-64, doi: http://dx.doi.org/10.1016/0277-3791(88)90093-5.

Mylroie, J., and J. Carew (1995), Karst development on carbonate islands, AAPG Special Volumes, pp. 55-76.

Neufeld, J. A., M. A. Hesse, A. Riaz, M. A. Hallworth, H. A. Tchelepi, and H. E. Huppert (2010), Convective dissolution of carbon dioxide in saline aquifers, Geophys. Res. Lett., 37(22), L22,404, doi:10.1029/2010GL044728.

Neuman, S., and D. Tartakovsky (2009), Perspective on theories of non-fickian transport in heterogeneous media, Adv. Water Resour., 32(5), $670-680$, doi: http://dx.doi.org/10.1016/j.advwatres.2008.08.005, dispersion in Porous Media. 
Okubo, A. (1970a), Horizontal dispersion of floatable particles in the vicinity of velocity singularities such as convergences, Deep Sea Research and Oceanographic Abstracts, 17(3), 445 - 454, doi:http://dx.doi.org/10.1016/0011-7471(70)90059-8.

Okubo, A. (1970b), Horizontal dispersion of floatable particles in the vicinity of velocity singularities such as convergences, Deep Sea Res. Oceanogr. Abstr, 17, 207 - 254.

Ottino, J. (1989), The Kinematics of Mixing: Stretching, Chaos, and Transport, Cambridge Texts in Applied Mathematics, Cambridge University Press.

Parkhurst, D. L. (1995), A compute program for speciation, reaction-path, advectivetransport, and inverse geochemical calculations, Tech. rep., U.S. Geological Survey Water-Resources Investigations Report, Lakewood, CO.

Pedretti, D., D. Fernández-Garcia, D. Bolster, and X. Sanchez-Vila (2013), On the formation of breakthrough curves tailing during convergent flow tracer tests in threedimensional heterogeneous aquifers, Water Resour. Res, 49(7), 4157-4173, doi: 10.1002/wrcr.20330.

Plummer, L. (1975), Mixing of sea water with calcium carbonate groundwater., Geol. Sot. Am. Mem., 142, 219-236.

Pool, M., V. Post, and C. Simmons (2015), Effects of tidal fluctuations and spatial heterogeneity on mixing and spreading in spatially heterogeneous coastal aquifers, Water Resources Research, 51(3), 1570-1585, doi:10.1002/2014WR016068.

Pool, M., M. Dentz, and V. Post (2016), Transient forcing effects on mixing of two fluids for a stable stratification, Water Resources Research, 52(9), 7178 - 7197, doi: 10.1002/2016WR019181.

Pope, S. B. (2000), Tubulent Flows, Cambridge University Press.

Rehfeldt, k., and L. Gelhar (1992), Stochastic analysis of dispersion in unsteady flow in heterogeneous aquifers, Water Resour. Res., 28, 2085 - 2099.

Renard, P., and D. Allard (2013), Connectivity metrics for subsurface flow and transport, Advances in Water Resources, 51, 168-196, doi: http://dx.doi.org/10.1016/j.advwatres.2011.12.001, 35th Year Anniversary Issue.

Rezaei, M., E. Sanz, E. Raeisi, C. Ayora, E. Vazquez-Sune, and J. Carrera (2005), Reactive transport modeling of calcite dissolution in the fresh-salt water mixing zone, $J$. Hydrol., 311(1), 282-298, doi:http://dx.doi.org/10.1016/j.jhydrol.2004.12.017.

Rubin, Y. (2003), Applied stochastic hydrology, Oxford Univ. Press, New York. 
Sanford, W., and L. Konikow (1989), Simulation of calcite dissolution and porosity changes in saltwater mixing zones in coastal aquifers, Water Resources Research, 25(4), 655-667, doi:10.1029/WR025i004p00655.

Schincariol, R., and F. Schwartz (1990), An experimental investigation of variable density flow and mixing in homogeneous and heterogeneous media, Water Resour. Res., 26(10), $2317-2329$.

Schincariol, R., F. Schwartz, and C. Mendoza (1997), Instabilities in variable density flows: stability and analysis for homogeneous and heterogeneous media, Water Resour. Res., 33(1), 31-41.

Schincariol, R. A. (1998), Disperse mixing dynamics of dense miscible plumes: natural perturbation initiation by local-scale heterogeneities, J. Contam. Hydrol., 34, 247-271.

Schotting, R., H. Moser, and S. HassMoser (1999), High-concentration-gradient dispersion in porous media: experiments, analysis and approximations, Adv. Water Resour., 22(7), 665-680, doi:http://dx.doi.org/10.1016/S0309-1708(98)00052-9.

Schwarz, C. (1999), Dichteabhängige strömungen in homogenen und heterogenen porösen medien., dissertation, Eidg. Tech. Hochsch. Zu-rich, Zurich, Switzerland.

Simmons, C. T., T. R. Fenstemaker, and J. M. J. Sharp (2001), Variable-density groundwater flow and solute transport in heterogeneous porous media: approaches, resolutions and future challenges, J. Contam. Hydrol, 52, 245-275.

Singurindy, O., B. Berkowitz, and R. Lowell (2004), Carbonate dissolution and precipitation in coastal environments: Laboratory analysis and theoretical consideration, Water Resour. Res., 40(4), doi:10.1029/2003WR002651, w04401.

Smart, P., J. Dawans, and F. Whitaker (1988), Carbonate dissolution in a modern mixing zone, Nature, 335(6193), 811-813, doi:10.1038/335811a0.

Stoessell, R., W. Ward, B. Ford, and J. Schuffert (1989), Water chemistry and caco3 dissolution in the saline part of an open-flow mixing zone, coastal yucatan peninsula, mexico, Geol. Soc. Am. Bull., 101, 159-1969.

Stoessell, R., Y. Moore, and J. Coke (1993), The occurrence and effect of sulfate reduction and sulfide oxidation on coastal limestone dissolution in yucatan cenotes, Ground Water, 31(4), 566-575, doi:10.1111/j.1745-6584.1993.tb00589.x.

Stoessell, R. K. (1995), Dampening of transverse dispersion in the halocline in karst limestone in the northeastern yucatan peninsula, GroundWater, 33(3), 366-371, doi: 10.1111/j.1745-6584.1995.tb00291.x. 
Tartakovsky, A. M., G. Redden, P. C. Lichtner, T. D. Scheibe, and P. Meakin (2008),

Mixing-induced precipitation: Experimental study and multiscale numerical analysis, Water Resour. Res., 44, W06S04.

Tennekes, H., and J. L. Lumley (1972), A First Course in Turbulence, MIT

Press,Cambridge,Mass.

Townley, L. R. (1995), The response of aquifers to periodic forcing, Adv. Water Resour., 18(3), 125-146, doi:10.1016/0309-1708(95)00008-7.

Tyukhova, A., and M. Willmann (2016), Connectivity metrics based on the path of smallest resistance, Adv. Water Resour., 88, 14-20, doi: https://doi.org/10.1016/j.advwatres.2015.11.014.

Tyukhova, A., W. Kinzelbach, and M. Willmann (2015), Delineation of connectivity structures in 2-d heterogeneous hydraulic conductivity fields, Water Resour. Res., 51(7), 5846-5854, doi:10.1002/2014WR015283.

Van Der Kamp, G. S. (1972), Tidal fluctuations in a confined aquifer extending under the sea, Proc. lnt. Geol. Congr., 24th, 101-106.

Villermaux, E. (2012), Mixing by porous media, C. R. Mécanique, 340, 933-943.

Voss, C. (1984), A finite-element simulation model for saturatedunsaturated, fluid-densitydependent ground-water flow with energy transport or chemically reactive single-species solute transport., US Geological Survey, Virginia.

Voss, C. I., and A. Provost (2002), SUTRA, a model for saturated-unsaturated variabledensity ground-water flow with solute or energy transport, Water-Resources Investigations Report 02-4231, U.S. Geological Survey.

Weiss, J. (1991), The dynamics of enstrophy transfer in two-dimensional hydrodynamics, Physica D, 48, $273-294$.

Williams, P. (1977), Hydrology of the waikoropupu springs: a major tidal karst resurgence in northwest nelson (new zealand), J. Hydrol., , 35, 73-92.

Willmann, M., J. Carrera, and X. SÃąnchez-Vila (2008), Transport upscaling in heterogeneous aquifers: What physical parameters control memory functions?, Water Resour. Res., 44(12), doi:10.1029/2007WR006531, w12437.

Zech, A., S. Attinger, V. Cvetkovic, G. Dagan, P. Dietrich, A. Fiori, Y. Rubin, and G. Teutsch (2015), Is unique scaling of aquifer macrodispersivity supported by field data?, Water Resour. Res., 51, doi:10.1002/2015WR017220. 
988

989

990

991

Zinn, B., and C. F. Harvey (2003), When good statistical models of aquifer heterogeneity go bad: A comparison of flow, dispersion, and mass transfer in connected and multivariate gaussian hydraulic conductivity fields, Water Resour. Res., 39(3), doi: 10.1029/2001WR001146, 1051. 